\title{
Superresolution microscopy reveals partial preassembly and subsequent bending of the clathrin coat during endocytosis
}

Markus Mund*1,2, Aline Tschanz*1,3, Yu-Le $\mathrm{Wu}^{1,3}$, Felix Frey ${ }^{4}$, Johanna L. Mehl ${ }^{1,5}$, Marko Kaksonen ${ }^{2,6}$, Ori Avinoam ${ }^{1,7}$, Ulrich S. Schwarz ${ }^{8,9}$, Jonas Ries ${ }^{1, \#}$

${ }^{1}$ European Molecular Biology Laboratory, Cell Biology and Biophysics, Meyerhofstrasse 1, 69117 Heidelberg, Germany.

2 University of Geneva, Department of Biochemistry, Quai Ernest-Ansermet 30, 1205 Geneva, Switzerland.

${ }^{3}$ Candidate for Joint PhD Programme of EMBL and University of Heidelberg.

${ }^{4}$ Kavli Institute of Nanoscience, Department of Bionanoscience, Delft University of Technology, Van der Maasweg 9, 2629 HZ Delft, The Netherlands.

${ }^{5}$ Present address: ETH Zurich, Switzerland.

${ }^{6}$ University of Geneva, NCCR Chemical Biology, Geneva, Switzerland.

${ }^{7}$ Weizmann Institute of Science, Department of Biomolecular Sciences, Herzl St 234, Rehovot, Israel.

${ }^{8}$ Institute for Theoretical Physics and Bioquant, Heidelberg University, Philosophenweg 19, 69120 Heidelberg, Germany.

${ }^{9}$ Bioquant, Heidelberg University, Im Neuenheimer Feld 267, 69120 Heidelberg, Germany.

* equal contribution

\# Correspondence: Jonas.ries@embl.de

\section{Summary}

Eukaryotic cells use clathrin-mediated endocytosis to take up a large range of extracellular cargos. During endocytosis, a clathrin coat forms on the plasma membrane, but it remains controversial when and how it is remodeled into a spherical vesicle.

Here, we use 3D superresolution microscopy to determine the precise geometry of the clathrin coat at endocytic sites. Through pseudo-temporal sorting, we determine the average trajectory of clathrin remodeling during endocytosis and find that clathrin coats assemble first on flat membranes to $\approx 50 \%$ of the coat area, before they become rapidly and continuously bent. We introduce a mathematical model that assumes a positive feedback for curvature generation of the clathrin coat. The cooperative curvature model agrees excellently with experimental data in three cell lines, and likely describes a general pathway for clathrin coat remodeling during endocytosis. 


\section{Introduction}

Endocytosis is an essential function of eukaryotic cells to internalize molecules from their surface. The major endocytic pathway is clathrin-mediated endocytosis (CME), which supports the uptake of many diverse cargos including nutrients, signaling molecules, membrane proteins, and pathogens. During CME, a dense coat of proteins self-assembles on the inner leaflet of the plasma membrane. The membrane itself is bent into an $\Omega$-shaped invagination that is eventually pinched off to form a coated vesicle, completing endocytosis (Kaksonen and Roux, 2018).

The major component of the coat are clathrin triskelia, which comprise three heavy and three light chains (Fotin et al., 2004). When triskelia are bound to the plasma membrane by adaptor proteins, they form ordered clathrin lattices. The structural flexibility of triskelia allows these lattices to adapt variable ratios of predominantly pentagonal and hexagonal faces, forming both flat and curved geometries.

Both geometries have been observed in vivo and in vitro, and their structure has been well characterized in vitro from a structural biology perspective (Cheng et al., 2007; Dannhauser and Ungewickell, 2012; Fotin et al., 2004; Heuser and Kirchhausen, 1985; Morris et al., 2019; Pearse, 1976; Smith et al., 1998; Takei et al., 1998; Ungewickell and Branton, 1981). However, it remains elusive how clathrin coat formation and membrane bending are temporally and causally related during endocytosis in cells.

Two main models of clathrin coat formation during endocytosis have been put forward. In the constant area model, clathrin grows to its final surface area as a flat coat, which then becomes continuously more curved until vesicle formation is complete. This model was based on the observation of clathrin lattices with many different curvatures in EM micrographs, and the assumption that all clathrin structures are endocytic intermediates (Heuser, 1980). Later, it was observed that flat clathrin lattices are mostly hexagonal. It was reasoned that for flat hexagonal lattices to become spherical, at least 12 pentagons have to be integrated, requiring extensive lattice remodeling (Kirchhausen, 1993). This was deemed thermodynamically and structurally unfavorable and thus unlikely to occur, leading to the formulation of a constant curvature model, which assumes that flat clathrin structures are not endocytic precursors. Instead, it was proposed that the endocytic clathrin coat assumes its final curvature, i.e. the curvature of the vesicle, directly from the start, and continuously grows in surface area over time at constant coat curvature.

The constant curvature model has been prevalent in the field, but recently challenged by reports that flat clathrin coats can indeed change curvature during endocytosis (Avinoam et al., 2015; Bucher et al., 2018; Scott et al., 2018). This has once again boosted interest in clathrin remodeling (Chen and Schmid, 2020; Kaksonen and Roux, 2018; Sochacki and Taraska, 2018). The last years have seen numerous studies based on diverse methods that did not converge on a common result. Reports supported either the constant area model (Avinoam et al., 2015; Sochacki et al., 2021), the constant curvature model (Willy et al., 2019), a combination of both models (Bucher et al., 2018; Tagiltsev et al., 2021; Yoshida et al., 2018) 
or the simultaneous existence of both models within cells (Scott et al., 2018). Thus, our current understanding of endocytic clathrin coat formation remains intriguingly inconclusive and incomplete.

Here, we present a quantitative survey of the three-dimensional (3D) clathrin coat architecture at endocytic sites that covers the whole timeline of endocytosis. We developed a powerful superresolution microscopy pipeline to acquire thousands of high-resolution, 3D snapshots of endocytic clathrin structures in three different cell lines. We used a novel model fitting framework to extract geometric parameters that allowed us to sort these snapshots according to their progression along the endocytic timeline. Thereby, we were able to infer endocytic dynamics from images of fixed cells, and to reconstruct the stereotypic remodeling of clathrin during endocytosis at the nanoscale.

In detail, we found that a clathrin coat first partially assembles on a flat membrane, and then simultaneously grows in surface area and coat curvature. While initial bending occurs rapidly, it later slows down and endocytic sites are eventually paused in a state of high curvature before vesicle scission. This trend is conserved across cell lines, suggesting it is a common attribute that is not affected by cell type.

Based on our data, we were able to develop a new kinetic growth model, the cooperative curvature model. In this model, clathrin triskelia are added to the edge of the growing clathrin coat, but at the same time, the tendency to bend the bulk of the growing coat also increases due to a positive feedback in curvature generation. The cooperative curvature model can be solved analytically and shows excellent agreement with the experimental data. This suggests that while single triskelia provide the required preferred curvature, only their interplay in a lattice provides the required physical force to bend the membrane.

\section{Results}

\section{Quantitative 3D superresolution imaging of clathrin structures}

Here, we use 3D single-molecule localization microscopy (SMLM) to systematically determine the precise geometry of individual clathrin-coated structures at the plasma membrane. For this, we optimized the sample preparation to densely label clathrin at endocytic sites, which is crucial to achieve high quality SMLM (Mund and Ries, 2020). We then localized sparsely activated single fluorophores by fitting an experimentally derived model of the astigmatic point-spread function using a method we developed recently (Li et al., 2018). This improved the resolution to $\approx 15 \mathrm{~nm}$ in $\mathrm{x} / \mathrm{y}$ (FWHM) and $\approx 30 \mathrm{~nm}$ in $\mathrm{z}$, and reduced typically observed image distortions. The achieved image quality allowed us to clearly visualize and quantify the 3D clathrin coat shape at different stages of endocytic site maturation (Figure 1A).

The large majority of sites were single structures that were well-isolated from each other, and exhibited great structural diversity. In addition, we noted several clusters of closely juxtaposed endocytic sites (Supplementary Figure 1A). In the isolated sites, we observe a 
variety of shapes including flat, curved, dome-shaped and spherical structures of different sizes (Figure 1A), indicating that endocytosis has been arrested by fixation at different time points during endocytic progression.

To quantify size and shape of individual clathrin coats, we used a computational pipeline based on a maximum-likelihood model fitting framework that we developed previously (Wu et al., 2021). This framework directly fits 3D geometric models to the 3D point cloud of localizations (Figure 1B) instead of rendered images, and thereby harnesses the full information contained in the SMLM data, including for instance the localization precision, rather than just spatial coordinates.

We describe the clathrin coat as a sphere segment that is defined by a radius $r$ and a closing angle $\theta$ (Figure 1C). Our model also accounted for coat thickness, antibody size and blurring due to the localization precision (Methods). Hence, it describes both shallow and highly curved structures equally well. Moreover, because $\theta$ increases during endocytosis from $0^{\circ}$ at flat sites to $180^{\circ}$ at complete vesicles (Figure $1 \mathrm{~B}$ ), we could use this parameter to sort the individual images according to endocytic progress (Figure 1D).

(A) 3D SMLM to visualize cellular clathrin-coated structures

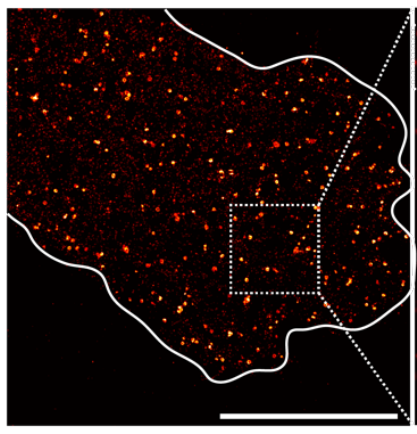

(B)

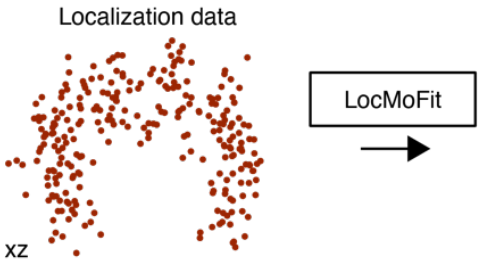

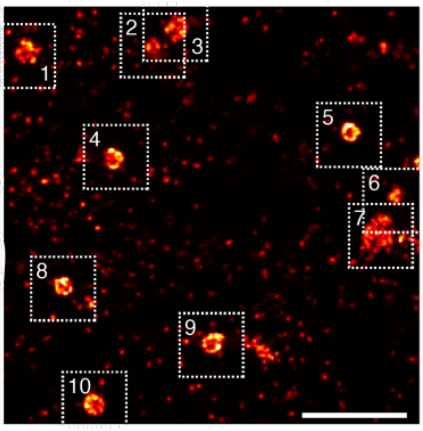

(C)

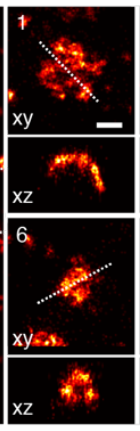

(D)
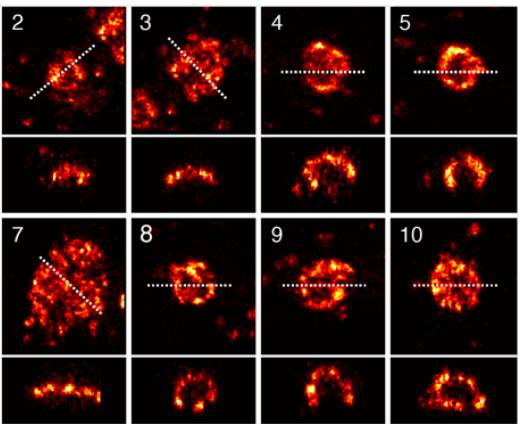

Geometric description
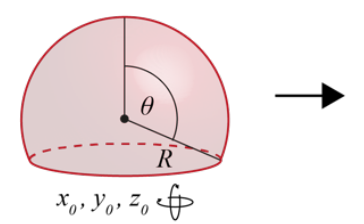

Identification of relative timepoint

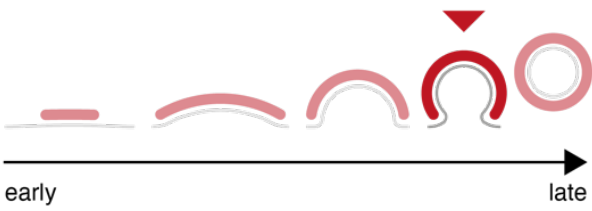

Figure 1-3D SMLM of clathrin-coated structures. (A) 3D SMLM image of immunolabeled clathrin at the bottom membrane of a SK-MEL-2 cell. The 3D geometry of clathrin-coated structures is clearly resolved, and exhibits great variability representing different timepoints of endocytosis. Top view with dotted line (xy) indicating 50 nm-thick z-slices (xz) are shown for 10 example sites. Scale bars are $10 \mu \mathrm{m}$ (left), $1 \mu \mathrm{m}$ (middle), $100 \mathrm{~nm}$ (right) (B) Systematic geometric analysis pipeline. All clathrin coats are segmented, and their localizations are directly fitted with a spherical cap model using the fitting framework LocMoFit (Wu et al., 2021). (C) In LocMoFit, individual clathrin coats are parametrized by their size (radius $R$ ), closing angle $(\theta)$, position $\left(x_{0}, y_{0}, z_{0}\right)$ and $3 D$ orientation. $(D)$ Using $\theta$ as a proxy for endocytic progression, the relative endocytic time points for each structure can be determined. 


\section{The clathrin coat grows in area and becomes more curved during endocytosis}

We first imaged immunostained clathrin in chemically fixed SK-MEL-2 cells, where we focused on the bottom plasma membrane that was densely covered by endocytic sites. These cells have been extensively studied and are established model cells for clathrin-mediated endocytosis with well-characterized endocytic dynamics (Aguet et al., 2013; Avinoam et al., 2015; Doyon et al., 2011; Kaplan et al., 2020). Using the 3D model fitting pipeline, we determined radius $R$, closing angle $\theta$, position and rotation of 1798 endocytic sites from 13 cells (Figure 2A). From the distribution of $\theta$, we found that two structural intermediates are enriched, and others are rare (Figure $2 \mathrm{~B}$ ). Only a small fraction of sites were completely flat. Slightly curved sites with $\theta \approx 70^{\circ}$ and strongly curved sites with $\theta \approx 130^{\circ}$ were enriched, indicating that those structural states are more frequently found in cells. We only rarely obtained sites with $\theta \approx 180^{\circ}$, which corresponds to a complete spherical coat, even though fully formed vesicles are found in our data (Supplementary Figure 1B). This indicates that at the time point of scission, the clathrin coat of nascent vesicles is still incomplete at the neck. Curvatures $H=1 / R$ ranged from 0 to $0.022 \mathrm{~nm}^{-1}$ with a median of $0.011 \mathrm{~nm}^{-1}$, corresponding to a median radius of $87 \mathrm{~nm}$, and surface areas ranged from $9,000 \mathrm{~nm}^{2}$ to $140,000 \mathrm{~nm}^{2}$ with a median of $54,000 \mathrm{~nm}^{2}$. These values agree well with previous measurements of the vesicular coat using EM ((Avinoam et al., 2015), median curvature $0.015 \mathrm{~nm}^{-1}$, median surface 54,500 $\mathrm{nm}^{2}$ ).

We then wanted to understand how the clathrin coat geometry changes during endocytosis. Thus, we used the closing angle parameter $\theta$ to sort endocytic sites relative to each other in time. Irrespective of whether endocytosis follows a constant curvature or constant area model, $\theta$ monotonically increases from early to late endocytic time points (Figure $2 \mathrm{C}$ ), and can thus be used as unbiased proxy for endocytic progression (Avinoam et al., 2015).

The curvature $H$ was strongly correlated with $\theta$, indicating that coat curvature increases continuously during endocytosis (Figure 2D). Similarly, the surface area increased from 32,000 $\mathrm{nm}^{2}$ (median of $5 \%$ of sites with smallest theta) to $50,000 \mathrm{~nm}^{2}$ (median of $5 \%$ of sites with highest theta). The projected area decreased from $31,000 \mathrm{~nm}^{2}$ to $13,000 \mathrm{~nm}^{2}$ (median of $5 \%$ of sites with lowest and highest theta respectively), which is slightly larger for flat sites, but in close agreement with previous EM measurements (Bucher et al., 2018). It is readily obvious that our data are incompatible with the constant curvature model, as the curvature is not constant, but increases monotonically with $\theta$. Just as clearly, our data do not support the constant area model, because the coat surface also increases during endocytosis.

Almost all data points are part of one continuous point cloud (Figure 2D), indicating a continuous transition between structural states during endocytosis. We noticed an additional, small disconnected set of data points representing $8.5 \%$ of all sites that correspond to endocytic sites with curvatures above $0.016 \mathrm{~nm}^{-1}$ and $\theta$ of $80^{\circ}-180^{\circ}$ (Figure 2D, Supplementary Figure 2), which likely represent small clathrin-coated vesicles from the trans Golgi. 


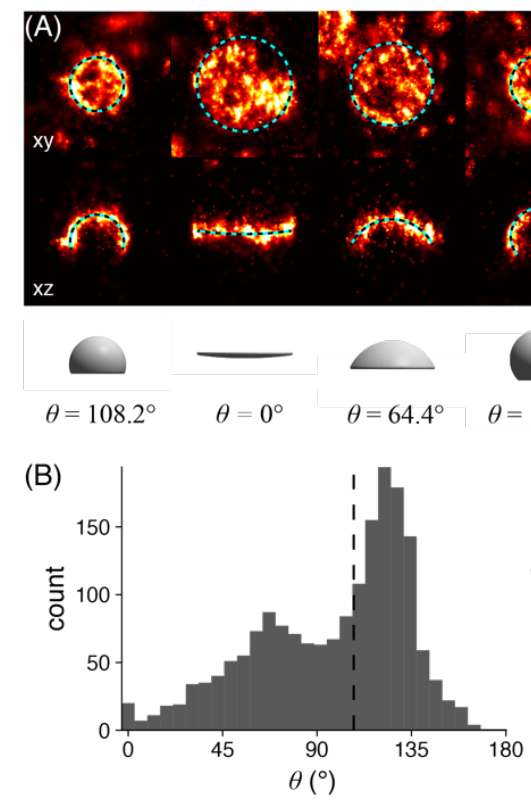

(C) Constant area model

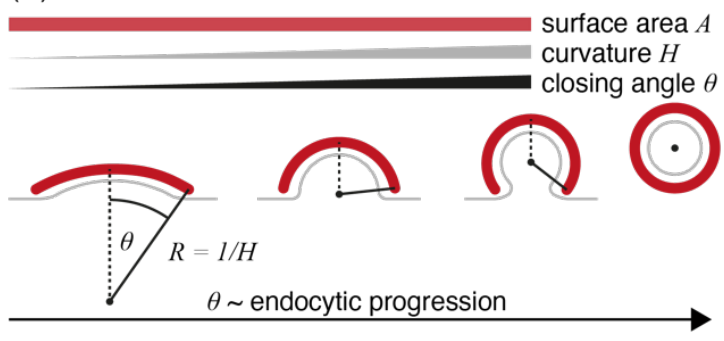

(D)
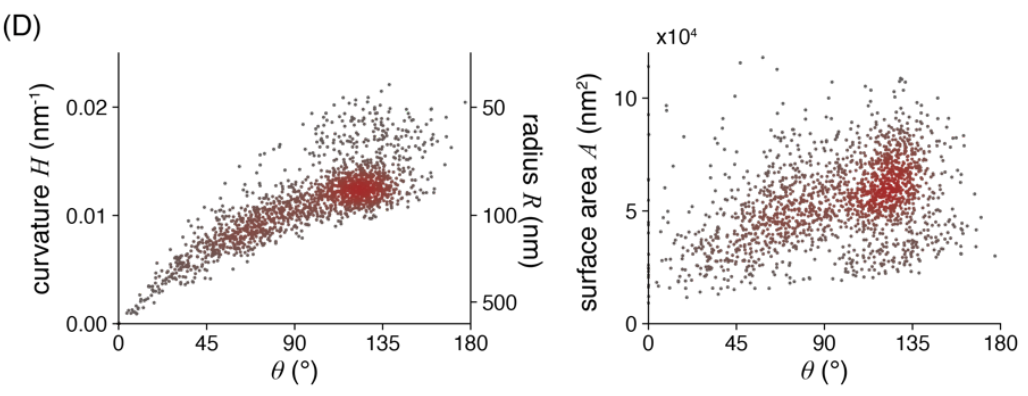

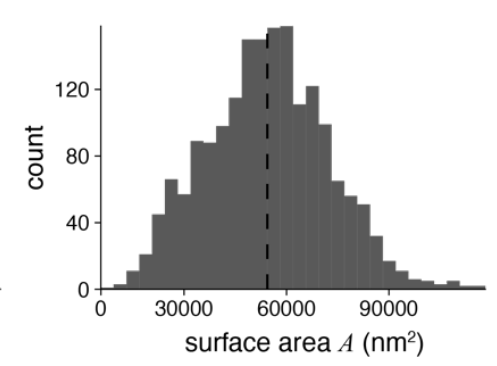

Constant curvature model
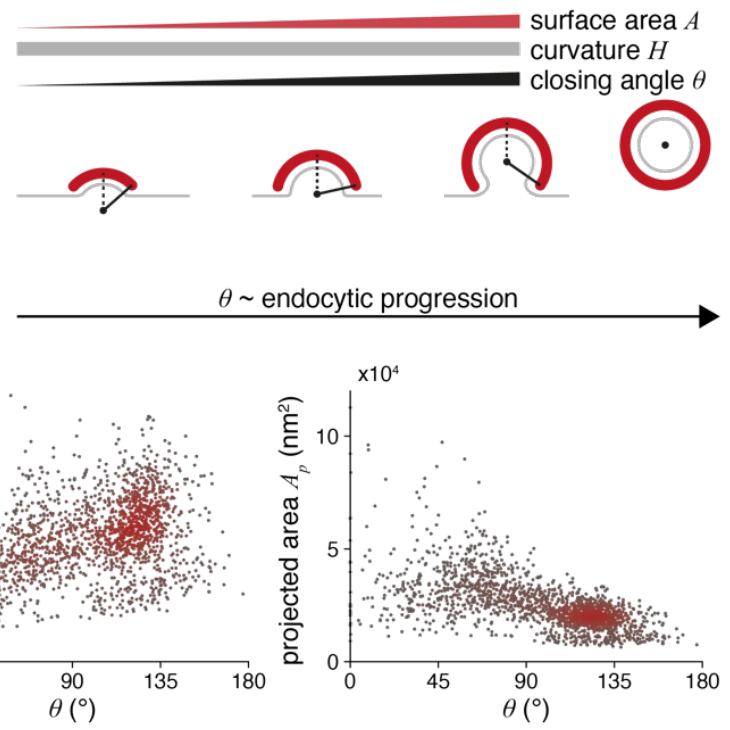

Figure 2 - Quantitative analysis of clathrin-coated structures in SK-MEL-2- cells. (A) Clathrin coat geometry is quantified using LocMoFit. The fitted spherical cap model is drawn as circle with its outer radius (top row, $x y$ ), as cross-sectional profile (50 $\mathrm{nm} x \mathrm{x}$ slices, middle row), and as surface with the corresponding closing angle $\theta$ (bottom row). Scale bar $100 \mathrm{~nm}$. (B) Distributions of closing angle $\theta$ (median $=108^{\circ}$ ), curvature $H($ median $=$ $0.011 \mathrm{~nm}^{-1}$ ) and surface area $A$ (median $=54,000 \mathrm{~nm}^{2}$ ) of endocytic sites in SK-MEL-2 cells ( $n=1798$ sites, $N=13$ cells) as determined from the model fit. (C) Two previously proposed mechanistic models for clathrin coat assembly during endocytosis (for details see text). In both scenarios $\theta$ increases monotonically and is thus a proxy for endocytic progression. (D) Development of curvature, surface area and projected area of the clathrin coat during endocytosis. Color indicates point density. 


\section{The cooperative curvature model of clathrin coat remodeling}

Our data clearly showed that clathrin coats grow and become more curved as the plasma membrane gets bent to produce a vesicle. Because the data quantitatively describes all 3D geometries that the endocytic clathrin coat assumes, it allowed us to move towards a mathematical model of clathrin coat formation during endocytosis.

We developed a kinetic model of clathrin coat growth based on basic knowledge about the structure and dynamics of clathrin coats (Figure 3A). We assume that the clathrin coat starts growing on a flat membrane. While triskelia have been shown to exchange dynamically during endocytosis (Avinoam et al., 2015), we assume that net growth of the area $A$ occurs via the addition of triskelia at the lattice edge $\mathcal{E}$ with a constant growth rate $k_{\text {on }}$ (Equation 1 ).

$$
\frac{d}{d t} A=\dot{A}=k_{\mathrm{on}} \mathcal{E}
$$

Moreover, we assume that the intrinsic pucker angle of individual triskelia and their interactions in a lattice together translate into an overall preferred curvature $H_{0}$ of the clathrin coat as a whole. However, the initially flat assembly suggests that this preferred curvature cannot be realized in an immature lattice. We hypothesize that cooperative processes in the coat are required to let the curvature grow. Coat curvature $H$ then increases asymptotically towards $H_{0}$ at an initial rate $\gamma$, slowing down towards zero when the preferred curvature is reached (Equation 2).

$$
\frac{d}{d \theta} H=\gamma\left(1-\frac{H^{2}}{H_{0}^{2}}\right)
$$

Choosing a quadratic dependence of the rate of curvature change on curvature is the simplest way to represent cooperativity in the lattice, which recently has been demonstrated in experiments (Sochacki et al., 2021; Zeno et al., 2021). Equation 2 can be solved analytically to yield an expression of the curvature $H$ depending on $\theta$ (Equation 3 ), which can then be fitted to our data.

$$
H(\theta)=H_{0} \tanh \left(\frac{\gamma \theta}{H_{0}}\right)
$$

Analogous expressions for other geometric parameters like surface area can be derived straightforwardly. A full description of the model is included in the Supplementary Note.

The fit of this model to the data of curvature $H$ in relation to closing angle $\theta$ shows excellent agreement (Figure 3B). As comparison, we also fitted the constant area model that fits the curvature data slightly worse than our model, and the constant curvature model that agrees poorly with the data. From the curvature fits, we calculated the corresponding curves for surface area (Figure $3 \mathrm{C}$ ) and the edge length of the clathrin coat (Figure 3D). The edge length decreases monotonically and approaches zero at $\theta=180^{\circ}$, thereby stopping growth according to Equation 1. Both datasets again highlight the very close agreement of the model prediction with the experimental data. From the fit, we determined that invagination occurs when about half of the total coat area has grown $\left(A_{0}=0.51\right)$, and that the preferred curvature 
of a clathrin coat is $H_{0}=0.014 \mathrm{~nm}^{-1}$, corresponding to a radius of $R_{0}=72 \mathrm{~nm}$. The model yields nearly identical parameters when surface area or rim length are fitted instead of curvature (Table 1), highlighting its robustness.

We then decided to test if the observed mode of clathrin remodeling is specific for SKMEL-2 cells. For this, we analyzed the endocytic clathrin ultrastructure also in U2OS cells and 3T3 mouse fibroblasts (Supplementary Figure 3 and Table 2-3). In these cell lines, just like in SK-MEL-2 cells, the curvature as well as the surface area continuously increase throughout endocytosis. We observe that the preferred curvature of the clathrin coat is smaller in U2OS $\left(H_{0}=0.012 \mathrm{~nm}^{-1}, R_{0}=85 \mathrm{~nm}\right)$ and $3 \mathrm{~T} 3$ cells $\left(H_{0}=0.011 \mathrm{~nm}^{-1}, R_{0}=89.7 \mathrm{~nm}\right)$ compared to SK-MEL-2 $\left(H_{0}=0.014 \mathrm{~nm}^{-1}, R_{0}=72 \mathrm{~nm}\right)$. This suggests that the average size of vesicles formed in $\mathrm{CME}$ is cell line specific. The fraction of the surface area acquired on the flat membrane is very similar for all three cell lines, with U2OS derived sites initiating curvature at $A_{0}=0.52$ and 3T3 sites at $A_{0}=0.45$ (Table 2-3). Taken together, we have shown in several cell lines that clathrin coats neither grow via constant curvature or constant area pathways, but rather first grow flat and then acquired curvature and more surface area simultaneously, with a non-linear mode of curvature generation.
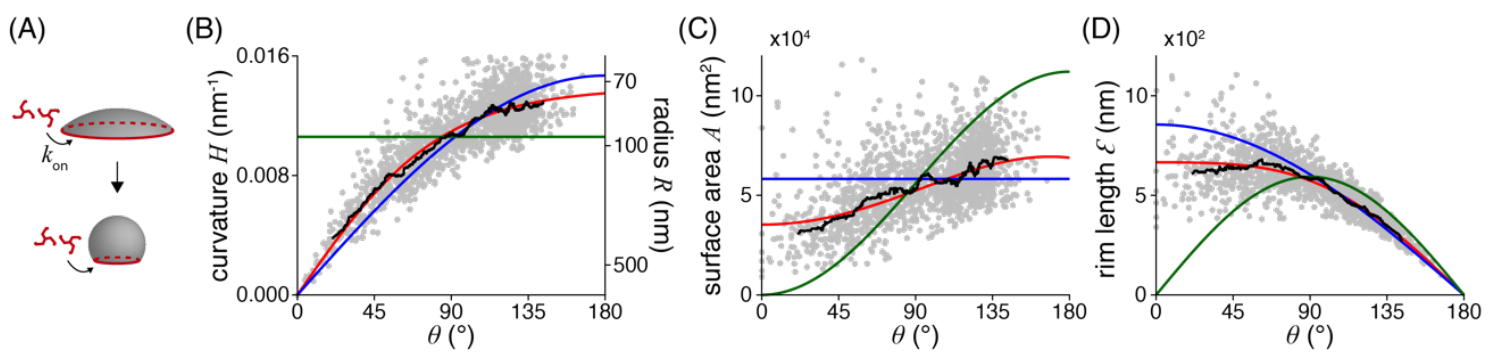

- cooperative curvature model

- constant curvature model

- constant area model

- rolling median

Figure 3 - Model for clathrin coat growth. (A) Schematic of the cooperative curvature model, where clathrin lattices grow by addition of triskelia to the rim at a constant growth rate $k_{\text {on }}$. Curvature generation is driven towards a preferred curvature, ultimately creating a spherical vesicle. (B) Distinct clathrin growth models and rolling median (window width $=82$ sites) fitted to curvature over $\theta$. The resulting fitting parameters are then used to map the same models also over $(C)$ surface area and $(D)$ rim length. ( $n=1631$ sites, $N=13$ cells).

\section{Temporal reconstruction of structural dynamics in endocytosis}

In our large dataset, we homogenously sample all endocytic time points, because we systematically segment all endocytic sites in the superresolution images. The distribution of structural states within the dataset is thus representative of their lifetime, with long-lived, stable curvature states being overrepresented in the data compared to transient states. This opens up the possibility to reconstruct the temporal progression of clathrin remodeling during endocytosis, and to ask whether clathrin coats acquire their curvatures with a constant rate, or if there are certain curvature transitions that occur more rapidly than others. 
For this, we sorted all endocytic sites by $\theta$. The rank of an endocytic site thus corresponds to its pseudotime, which describes its relative time point between 0 and 1 along the endocytic trajectory (Figure 4A).

As our model (Equation 1) describes the dynamic growth of the clathrin coat, we can solve it to derive an expression for $\theta$ over time $t$ (Equation 4), where the coat starts out flat and then starts to generate curvature, increasing $\theta$ over time.

$$
\theta(t)=\sqrt{\frac{24 \gamma k_{\mathrm{on}}}{8 \gamma^{2} H_{0}^{-2}-1} t}
$$

The square root dependence of $\theta$ on time $t$ reflects the slowing down of curvature generation as the clathrin coat approaches its preferred curvature. This expression fits the pseudotime-resolved data remarkably well (Figure 4B). Consistent with our previous reasoning, a linear model did not agree well with the data, since it gives a linear invagination for small $t$, emphasizing the validity of our cooperative curvature model, which leads to the characteristic square root dependence. The data slightly deviates from the model in the early phase, and more notably in the very end for pseudotimes close to 1 . This potentially indicates that clathrin geometry is influenced by other factors besides the coat itself close to vesicle scission.

Because this pseudotime resolved dataset was generated from a large number of endocytic sites in many cells, we effectively generated the average trajectories of how curvature, surface area, projected area and lattice edge change during endocytosis in SK-MEL2 cells (Figure 4B-E). We observe comparably few very flat clathrin coats. This shows that clathrin lattices are only transiently flat in the beginning of endocytosis for a very short time (Figure 4B), potentially because the initial bending may be mediated independently of clathrin coat (Zhao et al., 2017), e.g. by the action of BAR domain proteins (Henne et al., 2010), or at the base of filopodial projections.

Within the first $10 \%$ of pseudotemporal progression, the clathrin coat rapidly acquires shallow curvature of $H=0.007 \mathrm{~nm}^{-1}(R=134 \mathrm{~nm})$. They then become gradually more bent up to $\approx 60 \%$ of the endocytic timeline, when the sites reach an average curvature of $0.012 \mathrm{~nm}^{-1}$ $(R=83 \mathrm{~nm})$. During the last $\approx 40 \%$ of progression, the curvature increase almost stops, until vesicle scission occurs. Interestingly, the earliest sites in our dataset already contain $\approx 50 \%$ of the surface area of the final vesicles, which is also reflected in the fitting results $\left(A_{0}=0.51\right)$ (Figure 4E). This indicates that the initial assembly of the clathrin coat occurs very rapidly, or is in part below our detection limit. We performed identical analyses for U2OS and 3T3 derived clathrin sites and obtained highly similar results as for SK-MEL-2 cells, indicating that this trajectory represents a general pathway for clathrin coat remodeling during endocytosis (Supplementary Figure 4 and Tables 2, 3).

Finally, we made a 3D nanoscale movie, which vividly illustrates assembly and remodeling of the clathrin coat during endocytosis, from pseudotime resolved averages of our data. For this, we rescaled the radii of individual sites within each time bin to their median value, 
aligned them by their center coordinates and rotation angles, and computed the average clathrin coat shapes (Figure 4G). This yielded a nanoscale pseudo-temporal movie of endocytosis in SK-MEL-2 cells (Supplementary movie).

(A)

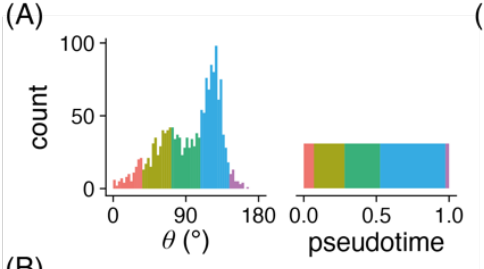

(B)

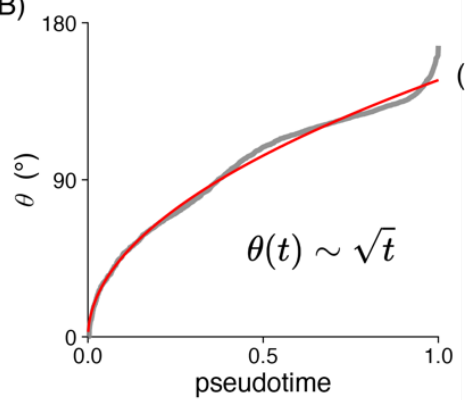

(C)

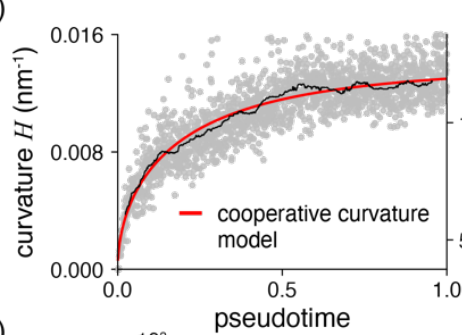

(D)

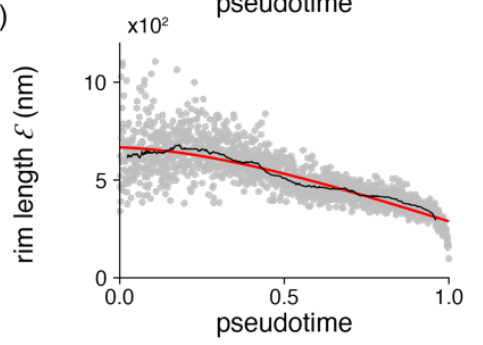

(E)

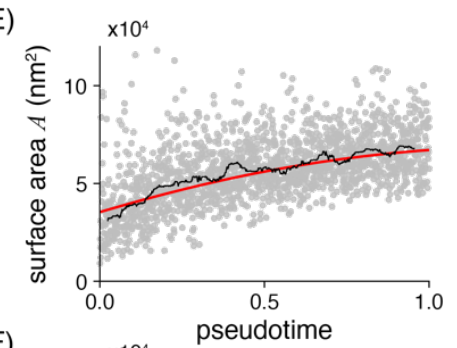

(F)

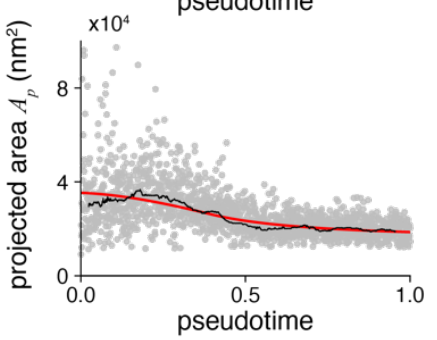

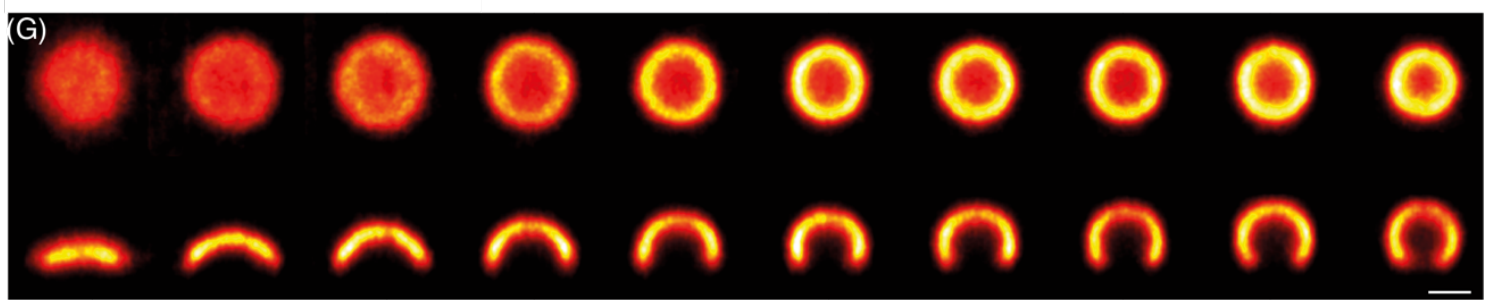

Figure 4 - Temporal reconstruction of clathrin coat remodeling. (A) Endocytic sites are sorted by $\theta$ to reconstruct pseudotime. Enriched $\theta$ states, for example the peak at $135^{\circ}$, represent long-lived states that remain for extended periods in pseudotime. Color code represents the same number of sites in both histograms. (B) The square-root dependence between $\theta$ and pseudotime approximated by the cooperative curvature model (red line). (C) Curvature generation over pseudotime is approximated by the cooperative curvature model. Fit results were used to describe (D) rim length, $(E)$ surface area and (F) projected area change over pseudotime. A rolling median (window of 82 sites) is plotted alongside (black line). (G) Superresolution averages for distinct endocytic stages, resulting from all collected snapshots. Each bin contains the same number of snapshots of clathrin-coated structures sorted along their pseudotime ( $n=163$ per bin), so that all bins represent equally long pseudotime intervals. Scale bar is $100 \mathrm{~nm}$. ( $n=1631$ sites, $N=13$ cells).

\section{Discussion}

\section{Quantitative description of clathrin coat ultrastructure}

The nature of clathrin assembly during endocytosis has become a classical question in cell biology that remains unresolved (reviewed in (Chen and Schmid, 2020; Sochacki and Taraska, 2018)). There have been two main competing mechanistic models of how triskelia form a coat: the constant curvature model predicts gradual assembly of triskelia, where the radius of the final vesicle is defined from the start of endocytosis. In contrast, the constant area model predicts that clathrin pre-assembles on a flat membrane before membrane and coat bending happen concomitantly until vesicle scission appears. 
Each model is supported by a number of studies, which mostly relied on electron microscopy techniques. Among them, 3D correlative electron tomography yields exquisite 3D endocytic membrane shapes (Avinoam et al., 2015), but can be tedious to extend towards large numbers of cells. Platinum replica electron microscopy offers large fields of view and thus superb throughput, but gives limited 3D resolution (Bucher et al., 2018; Sochacki et al., 2017).

We reasoned that the trajectory of clathrin coat assembly during endocytosis could best be determined by systematically imaging large numbers of endocytic sites with high 3D resolution. Therefore, we used SMLM that combines high 3D resolution with high throughput. Although lower than in electron microscopy, the resolution we achieved allowed us to resolve the precise 3D coat ultrastructure of clathrin-coated pits. Importantly, due to the molecular specificity for clathrin, we were able to unbiasedly segment all the endocytic sites at the bottom surface of the cells in our images, thus ensuring homogenous sampling of the structural variety of clathrin coats.

We applied a novel maximum-likelihood based fitting framework that we developed recently (Wu et al., 2021), which allows fitting complex geometric models to localization point clouds. Because the fit is applied directly to the localizations, it considers all quantitative parameters of all localizations, most notably localization uncertainty. This results in the precise and reliable quantification of the underlying structures (Wu et al., 2020).

We describe the clathrin coat as a spherical cap, which matches the large majority of sites very well. Additionally, in our data, we observe that some sites are asymmetric, ellipsoidal, or deformed more irregularly (Supplementary Figure $1 \mathrm{C}$ ). We do not currently evaluate this asymmetry. Rather, we introduce rotational symmetry during the averaging (Figure 4G), where we align each site based on their model parameters and thus average out many irregularities. A deviation of the cross-sectional profile from a circle is nevertheless preserved in the averaging (Supplementary Figure 5), and in future studies more complex geometric models will enable analyzing the structure and asymmetries of the coat in more detail.

\section{Distribution of clathrin coat curvatures}

In the first step of our analysis pipeline, we segment all clathrin-coated structures in the superresolution images. We thereby aim to achieve homogenous sampling to capture all endocytic intermediates with the same probability irrespective of time point, size, and orientation. Interestingly, the surfaces of the earliest endocytic sites are on average already half of the final surface. How do these earliest sites assemble? We cannot sort the very flat sites, which occur comparably rarely in our dataset, in time using $\theta$ because their $\theta$ is close to zero. However, their relative fraction among all sites is still very informative. Among all endocytic snapshots, short-lived states should be rarely found, while long-lived states will be observed frequently. Thus, the relative absence of small flat sites in our data indicates that the initial assembly occurs rapidly. 
In addition to the short lifetimes of small flat sites, two technical reasons could potentially contribute to their rare occurrence in our data. Due to their small size and potentially substoichiometric labeling, which is also noticeable as holes in larger structures (Supplementary Figure 1D), they might not have sufficient immunostaining signal and thus they might be hard to be differentiated from small clusters of primary and secondary antibodies that are routinely observed in SMLM images. Additionally, the very first small clathrin assemblies might be meta-stable, with mostly loosely bound triskelia, and thus might not be stabilized well by chemical fixation. It is interesting to note that very small sites are also rarely found in previously published electron microscopy datasets (Avinoam et al., 2015; Bucher et al., 2018; Sochacki et al., 2021), further indicating that these sites are short-lived.

Generally, completely flat clathrin coats are rare in our data. Instead, we observe that clathrin coats quickly acquire curvature already early in endocytosis, suggesting flat coats are not very stable. This notion is supported by recent demonstrations that flat clathrin coats store energy, which can drive coat bending without additional factors (Sochacki et al., 2021; Tagiltsev et al., 2021).

\section{The cooperative curvature model}

Here, we visualized the shapes of clathrin coats with high 3D resolution. This was crucial to precisely measure the clathrin curvature throughout the endocytic timeline ranging from flat to highly curved structures, as for instance flat and very shallow invaginations would appear identical in 2D imaging. Thus, our data enabled us to robustly test different growth models for clathrin during endocytosis.

The constant curvature model predicts a continuous increase in surface area, constant coat curvature, as well as a rim length that increases until a half spherical geometry is reached, and then decreases again. Our experimental data, most notably the increase in curvature and monotonical decrease in rim length, are incompatible with these predictions, thus ruling out the constant curvature model (Figure 3 , green lines).

The constant area model on the other hand predicts constant surface area, increasing curvature and decreasing rim lengths. These predictions agree reasonably well with the data for curvature and rim length, however fail to explain the monotonical increase in coat surface over time. We note that the surface area needs to grow also in the constant area model, which predicts that the coats assemble to the final size of a vesicle in the very beginning of endocytosis. Thus, the continuous increase of surface area that we observed here rule out the constant area model as well (Figure 3, blue lines).

Interestingly, we found that around half of the clathrin coat area has preassembled when plasma membrane invagination begins (Figure 2D, 4C), agreeing well with previous reports (Bucher et al., 2018; Scott et al., 2018), after which the coat keeps growing gradually. Based on this observation, we then developed a mathematical model that for the first time considers that curvature is generated in a positive (non-linear) feedback loop. The cooperative 
curvature model assumes that net area growth of the clathrin coat occurs via triskelia addition to the many available binding sites at the coat edge. We propose that this process depends on the number of free binding sites, which scale with the rim length, and can be described by a single kinetic constant $k_{\text {on }}$. Triskelia addition within the coat is nevertheless still likely to occur at some lower rate, as clathrin lattices can be unsaturated and have defects (Frey et al., 2020; Sochacki et al., 2021) and triskelia can exchange (Avinoam et al., 2015), which is energetically favorable for curvature changes within these lattices (Frey and Schwarz, 2020; Sochacki et al., 2021; Tagiltsev et al., 2021). While growth at the rim accounts for increase in surface area, curvature is most likely generated in the bulk of the coat. Here we assume a non-linear relation between rate of curvature increase and curvature, which reflects cooperativity in the lattice, e.g. due to rearrangements of neighboring triskelia or larger regions thereof. Only this assumption of cooperativity, which is supported by recent experiments (Sochacki et al., 2021; Zeno et al., 2021), gives the excellent fit between theory and experiment described above. Moreover, it predicts the square root law in time, which is a clear signature of a non-linear process.

Even though our model agrees with the data very well, it is still an incomplete description of the process, largely because it only considers the coat itself and ignores the plethora of other proteins within the endocytic machinery. These include numerous BAR domain proteins that get dynamically recruited and disassembled during endocytosis and thus are bound to influence membrane curvature at endocytic sites, as well as a variety of clathrin adaptor proteins, whose presence or absence could explain the cell-type specific differences in average vesicle sizes that we observed (Supplementary Figure 3). Taken together, these factors could explain the imperfection of our model in the very beginning, and the final part of the timeline (Figure 4A), where vesicle scission is driven by the mechanoenzyme dynamin that is recruited and acts rapidly. Additionally, we only modeled clathrin recruitment and ignored clathrin disassembly, which could be mediated by adaptor unbinding (Taylor et al., 2011) and uncoating factors including auxilin (He et al., 2020) that are recruited before the end of vesicle scission. Finally, we assume that clathrin coat has constant properties, most notably that the intrinsic coat-driven curvature generation towards its preferred curvature occurs unidirectionally and remains the same throughout endocytosis (equation 3 ). It is however likely that the properties of the clathrin coat change during endocytosis, e.g. by coat stiffening or increasingly tight interactions between triskelia (Frey and Schwarz, 2020; Frey et al., 2020; Sochacki et al., 2021).

Taken together, we characterized the dynamics and geometries of the clathrin coat in endocytosis by combining 3D superresolution microscopy, quantitative analysis and mathematical modeling. We found that clathrin bending and assembly occur simultaneously along a precisely defined trajectory. We anticipate that this work will be foundational to further study the structural mechanism of endocytosis, both under physiological and perturbed conditions, in mutant backgrounds, and in disease. 


\section{Acknowledgements}

We thank the entire Ries and Kaksonen labs for fruitful discussions and support. This work was supported by the European Research Council (ERC CoG-724489 to J.R.), the National Institutes of Health Common Fund 4D Nucleome Program (Grant U01 to J.R.), the Human Frontier Science Program (RGY0065/2017 to J.R.), the EMBL Interdisciplinary Postdoc Programme (EIPOD) under Marie Curie Actions COFUND (Grant 229597 to O.A.), the European Molecular Biology Laboratory (M.M., A.T., Y.-L. W. and J.R.) and the Swiss National Science Foundation (grant 310030B_182825 and NCCR Chemical Biology to MK). O.A is an incumbent of the Miriam Berman Presidential Development Chair.

\section{Author contributions}

M.M., O.A., M.K. and J.R. conceived the study, M.M., A.T., J.M. and O.A. performed experiments, M.M., A.T., Y.-L.W., J.M., O.A. and J.R. analyzed superresolution data; F.F. and U.S. developed the cooperative curvature model; J.R. supervised the study. M.M., A.T., F.F., J.R. wrote the manuscript with input from all authors.

\section{Declaration of interests}

The authors declare no competing interests. 


\section{Material and methods}

\section{Cell culture}

SK-MEL-2 cells (gift from David Drubin, UC Berkeley, described in (Doyon et al., 2011)) were cultured adherently as described previously (Li et al., 2018) in DMEM F12 (Dulbecco's modified Eagle's medium with Nutrient Mixture F-12) with GlutaMAX, phenol red (Thermo Fisher; 10565018), 10\% (v/v) FBS, ZellShield (Biochrom AG, Berlin, Germany), at $37^{\circ} \mathrm{C}$, under an atmosphere with 5\% CO2 and $100 \%$ humidity.

U2OS cells were cultured adherently as described previously (Thevathasan et al., 2019) in high-glucose DMEM supplemented with 10\% FBS, $2 \mathrm{mM}$ I-glutamine, non-essential amino acids, ZellShield at $37{ }^{\circ} \mathrm{C}$ under an atmosphere with $5 \% \mathrm{CO} 2$ and $100 \%$ humidity.

3T3 mouse fibroblasts (gift from Alba Diz-Muñoz, EMBL Heidelberg) were cultured adherently in DMEM (4.5 g/L D-Glucose) supplemented with 1× MEM NEAA (catalog no. 11140-035, Gibco), 1× GlutaMAX (catalog no. 35050-038, Gibco) and 10\% (v/v) fetal bovine serum (catalog no. $10270-106$, Gibco) at $37^{\circ} \mathrm{C}$ under an atmosphere with $5 \% \mathrm{CO}, 100 \%$ humidity.

\section{Sample preparation for superresolution imaging of clathrin-coated pits}

Cells were fixed as described previously (Li et al., 2018) using 3\% (w/v) formaldehyde 10 mM MES, pH 6.1, $150 \mathrm{mM} \mathrm{NaCl}, 5 \mathrm{mM}$ EGTA, $5 \mathrm{mM}$ d-glucose, $5 \mathrm{mM} \mathrm{MgCl} 2$ for $20 \mathrm{~min}$. Fixation was quenched in $0.1 \%(\mathrm{w} / \mathrm{v}) \mathrm{NaBH} 4$ for $7 \mathrm{~min}$. The sample was washed three times with PBS and permeabilized for $15 \mathrm{~min}$ with $0.01 \%(\mathrm{w} / \mathrm{v})$ digitonin (Sigma-Aldrich, St. Louis, $\mathrm{MO}, \mathrm{USA}$ ) in PBS. The sample was then washed twice with PBS and blocked for $1 \mathrm{~h}$ with $2 \%$ $(\mathrm{w} / \mathrm{v}) \mathrm{BSA} / \mathrm{PBS}$, washed with PBS, and stained for 3-12 $\mathrm{h}$ with anti-clathrin light chain (sc28276; Santa Cruz Biotechnology, Dallas, TX, USA) and anti-clathrin heavy chain rabbit polyclonal antibodies (ab21679; Abcam, Cambridge, UK) in 1\% (w/v) BSA/PBS. After three washes with PBS the sample was incubated for 3-4h with a secondary donkey anti-rabbit antibody (711-005-152; Jackson ImmunoResearch, West Grove, PA, USA) that was conjugated to Alexa Fluor 647-NHS at an average degree of labeling of 1.5. The sample was then washed three times and mounted for imaging in blinking buffer $(50 \mathrm{mM}$ Tris/ $\mathrm{HCl} \mathrm{pH} \mathrm{8,} 10 \mathrm{mM} \mathrm{NaCl}$, $10 \%(\mathrm{w} / \mathrm{v})$ D-glucose, $500 \mathrm{\mu g} \mathrm{ml}^{-1}$ glucose oxidase, $40 \mu \mathrm{g} \mathrm{ml}^{-1}$ glucose catalase, $35 \mathrm{mM}$ MEA in $\left.\mathrm{H}_{2} \mathrm{O}\right)$.

\section{Superresolution microscopy}

SMLM images were acquired at room temperature $\left(24^{\circ} \mathrm{C}\right)$ on a customized microscope (Mund et al., 2018) with a 160x NA 1.43 oil-immersion objective (Leica, Wetzlar, Germany). Illumination was done using a a LightHub laser combiner (Omicron-Laserage Laserprodukte, Dudenhofen, Germany) with Luxx 405, 488, and 638 Cobolt 561 lasers, which were triggered using a Mojo FPGA (Embedded Micro, Denver, CO, USA) for microsecond pulsing control of lasers. The laser was guided through a speckle reducer (LSR-3005-17S-VIS; Optotune, 
Dietikon, Switzerland) and coupled into a multimode fiber (M105L02S-A; Thorlabs, Newton, NJ, USA). The output of the fiber is magnified and imaged into the sample. Fiber generated fluorescence was removed using a laser clean-up filter (390/482/563/640 HC Quad; AHF, Tübingen, Germany). The focus was stabilized using a closed-loop system, based on reflecting the signal of a near-infrared laser by the coverslip and detecting the resulting signal on a quadrant photodiode, resulting in focus stabilities of $\pm 10 \mathrm{~nm}$ over several hours. Fluorescence emission was filtered using a 676/37 or a 700/100 bandpass filter (AHF) and recorded by an EMCCD camera (Evolve512D; Photometrics, Tucson, AZ, USA). Typically, 100,000-300,000 frames were acquired using 15-ms or 30ms exposure times and laser power densities of 15 $\mathrm{kW} / \mathrm{cm} 2$. 405-nm laser intensity was adjusted automatically by changing pulse duration in order to keep the number of localizations per frame constant during the acquisition.

\section{Data analysis}

All data analysis was conducted in SMAP ((Ries, 2020) based on MATLAB and available as open source under github.com/jries/SMAP).

\section{Superresolution image reconstruction}

For fitting the localizations, peaks were detected as maxima in reconstructed superresolution images after applying a difference-of-Gaussian filter. At those positions, cropped images of $13 \times 13$ pixels were fitted with an experimentally derived PSF model (free fitting parameters: $x, y, z$, photons per localization, background per pixel), using an MLE (Maximum likelihood estimation) fitter (Li et al., 2018). The $x, y$ and $z$ positions were corrected for residual drift by a custom algorithm based on redundant cross-correlation. Localizations persistent over consecutive frames (detected within $35 \mathrm{~nm}$ from one another and with a maximum gap of one dark frame) were merged into one localization by calculating the weighted average of $x, y$ and $z$ positions and the sums of photons per localization and background. Localizations were filtered by the localization precision in $x, y 0-20 \mathrm{~nm}$ to exclude dim localizations. Superresolution images were constructed with every localization rendered as a two-dimensional elliptical Gaussian with a width proportional to the localization precision (factor 0.4).

\section{Quantitative geometric analysis of clathrin-coated structures}

Clathrin-coated structures were segmented semi-automatically. First, we manually defined a region of interest excluding the edges of the cell. Then, the image was blurred using a Gaussian filter with a sigma of $100 \mathrm{~nm}$, and peaks were detected using a manually set threshold. This typically yielded several 100s of sites in a region of $\sim 30 \times 30 \mu \mathrm{m}$. These candidate sites were curated manually, and only single, well-isolated clathrin-coated structures were retained in the dataset.

Next, these structures were analyzed using LocMoFit, an MLE-based model fitting framework that we developed recently (Wu et al., 2021). LocMoFit directly fits localization 
coordinates with the probability density function (PDF) of a parametrized geometric model. In this study, we modeled clathrin-coated structures with a hollow spherical cap parameterized by the surface area $A$ and the closing angle $\theta . \theta$ is defined as the angle between the two vectors that point to the pole and to the rim, respectively, from the center of the sphere. The position of the model is defined as the center of mass of the cap. In practice, we discretized the cap by placing spiral points (Saff and Kuijlaars, 1997), or the spherical Fibonacci lattice, on the surface of the cap to approximate an even distribution. In LocMoFit, these points were treated as discrete fluorophore coordinates when constructing the PDF of the model. During fitting, additional parameters including the center position and the orientation of the model were determined with respect to the fluorophore coordinates, and an extra uncertainty and the background weight were applied to the PDF. After fitting the sphere radius is derived as $r=\sqrt{A / 2 \pi(1-\cos \theta)}$, projected area as $\pi \sin ^{2} \theta$ and edge length as $2 \pi \sin \theta$. For some flat sites where the fit produced slightly negative curvature values, curvature $\mathrm{H}$ and $\theta$ were set to $0 \mathrm{~nm}^{-1}$ and $0^{\circ}$ respectively in order to approximate them as completely flat.

After model fitting, a second curation step was performed. With this we ensured that only well fitted sites are included in the final data set. Sites were excluded if they were upside down (clearly originating from an upper membrane), double-sites with two sites clearly connected to each other or an adjacent flat structure, large plaques or structures nondistinguishable from an antibody cluster.

\section{Pseudo-temporal reconstruction of clathrin remodeling during endocytosis}

To sort endocytic sites in pseudotime, they were sorted by the closing angle $\theta$, assigning each site a rank index. Flat sites with a manually assigned $\theta=0$ were all assigned an index of 0 . As pseudo-temporal sorting assumes that all sites are part of the same endocytic trajectory, endocytic sites with curvatures above a cell line specific threshold $\left(H>0.016 \mathrm{~nm}^{-1}\right.$ for SKMEL-2; $\mathrm{H}>0.013 \mathrm{~nm}^{-1}$ for U2OS; $\mathrm{H}>0.014 \mathrm{~nm}^{-1}$ for $3 \mathrm{~T} 3$ ) that form a visibly disconnected point cloud (Supplementary Figures 2, 3) were excluded for this analysis. To compute pseudotemporal averages, the sites were spatially and rotationally aligned, and rescaled by the average radius of all sites within the respective bin. The supplementary movie was computed using a sliding window approach, where each frame shows the average of 30 sites, and the frame-to-frame increment is 20 sites. The median pseudotime of those 30 sites is indicated.

\section{Further data analysis}

All data that resulted from the quantitative geometric description of clathrin-coated structures were further analyzed in R ( $R$ Core Team, 2020). Fitting of the growth models (Tables 1-3) was performed on a filtered data set, excluding disconnected sites above a cell line specific threshold, and sites of negative curvature.

For depicting the growth models in Figure 3 and 4, as well as Supplementary Figure 3 and 4, parameters resulting from the $H(\theta)$ fit were used and mapped to the $A, \varepsilon$ and $A_{p}$ data. 


\section{References}

Aguet, F., Antonescu, C.N., Mettlen, M., Schmid, S.L., and Danuser, G. (2013). Advances in Analysis of Low Signalto-Noise Images Link Dynamin and AP2 to the Functions of an Endocytic Checkpoint. Developmental Cell 26, 279-291.

Avinoam, O., Schorb, M., Beese, C.J., Briggs, J.A.G., and Kaksonen, M. (2015). Endocytic sites mature by continuous bending and remodeling of the clathrin coat. Science 348, 1369-1372.

Bucher, D., Frey, F., Sochacki, K.A., Kummer, S., Bergeest, J.-P., Godinez, W.J., Kräusslich, H.-G., Rohr, K., Taraska, J.W., Schwarz, U.S., et al. (2018). Clathrin-adaptor ratio and membrane tension regulate the flat-to-curved transition of the clathrin coat during endocytosis. Nature Communications 9, 1109.

Chen, Z., and Schmid, S.L. (2020). Evolving models for assembling and shaping clathrin-coated pits. Journal of Cell Biology 219.

Cheng, Y., Boll, W., Kirchhausen, T., Harrison, S.C., and Walz, T. (2007). Cryo-electron Tomography of Clathrincoated Vesicles: Structural Implications for Coat Assembly. Journal of Molecular Biology 365, 892-899.

Dannhauser, P.N., and Ungewickell, E.J. (2012). Reconstitution of clathrin-coated bud and vesicle formation with minimal components. Nature Cell Biology 14, 634.

Doyon, J.B., Zeitler, B., Cheng, J., Cheng, A.T., Cherone, J.M., Santiago, Y., Lee, A.H., Vo, T.D., Doyon, Y., Miller, J.C., et al. (2011). Rapid and efficient clathrin-mediated endocytosis revealed in genome-edited mammalian cells. Nature Cell Biology 13, 331-337.

Fotin, A., Cheng, Y., Sliz, P., Grigorieff, N., Harrison, S.C., Kirchhausen, T., and Walz, T. (2004). Molecular model for a complete clathrin lattice from electron cryomicroscopy. Nature 432, 573-579.

Frey, F., and Schwarz, U.S. (2020). Competing pathways for the invagination of clathrin-coated membranes. Soft Matter.

Frey, F., Bucher, D., Sochacki, K.A., Taraska, J.W., Boulant, S., and Schwarz, U.S. (2020). Eden growth models for flat clathrin lattices with vacancies. New Journal of Physics 22, 073043.

He, K., Song, E., Upadhyayula, S., Dang, S., Gaudin, R., Skillern, W., Bu, K., Capraro, B.R., Rapoport, I., Kusters, I., et al. (2020). Dynamics of Auxilin 1 and GAK in clathrin-mediated traffic. Journal of Cell Biology 219.

Henne, W.M., Boucrot, E., Meinecke, M., Evergren, E., Vallis, Y., Mittal, R., and McMahon, H.T. (2010). FCHo Proteins Are Nucleators of Clathrin-Mediated Endocytosis. Science 328, 1281-1284.

Heuser, J. (1980). Three-dimensional visualization of coated vesicle formation in fibroblasts. Journal of Cell Biology 84, 560-583.

Heuser, J., and Kirchhausen, T. (1985). Deep-etch views of clathrin assemblies. Journal of Ultrastructure Research 92, 1-27.

Kaksonen, M., and Roux, A. (2018). Mechanisms of clathrin-mediated endocytosis. Nature Reviews Molecular Cell Biology 19, 313.

Kaplan, C., Kenny, S.J., Chen, S., Schöneberg, J., Sitarska, E., Diz-Muñoz, A., Xu, K., and Drubin, D.G. (2020). Adaptive actin organization counteracts elevated membrane tension to ensure robust endocytosis. BioRxiv 2020.04.05.026559.

Kirchhausen, T. (1993). Coated pits and coated vesicles - sorting it all out. Current Opinion in Structural Biology 3, 182-188.

Li, Y., Mund, M., Hoess, P., Deschamps, J., Matti, U., Nijmeijer, B., Sabinina, V.J., Ellenberg, J., Schoen, I., and Ries, J. (2018). Real-time 3D single-molecule localization using experimental point spread functions. Nature Methods 15, 367-369.

Morris, K.L., Jones, J.R., Halebian, M., Wu, S., Baker, M., Armache, J.-P., Avila Ibarra, A., Sessions, R.B., Cameron, A.D., Cheng, Y., et al. (2019). Cryo-EM of multiple cage architectures reveals a universal mode of clathrin self-assembly. Nature Structural \& Molecular Biology 26, 890-898.

Mund, M., and Ries, J. (2020). How good are my data? Reference standards in superresolution microscopy. MBoC 31, 2093-2096.

Mund, M., van der Beek, J.A., Deschamps, J., Dmitrieff, S., Hoess, P., Monster, J.L., Picco, A., Nédélec, F., Kaksonen, M., and Ries, J. (2018). Systematic Nanoscale Analysis of Endocytosis Links Efficient Vesicle Formation to 
bioRxiv preprint doi: https://doi.org/10.1101/2021.10.12.463947; this version posted October 13,2021 . The copyright holder for this preprint (which was not certified by peer review) is the author/funder, who has granted bioRxiv a license to display the preprint in perpetuity. It is made available under aCC-BY-NC-ND 4.0 International license.

Patterned Actin Nucleation. Cell 174, 884-896.e17.

Pearse, B.M. (1976). Clathrin: a unique protein associated with intracellular transfer of membrane by coated vesicles. Proc Natl Acad Sci USA 73, 1255.

$R$ Core Team (2020). R: A Language and Environment for Statistical Computing (Vienna, Austria: R Foundation for Statistical Computing).

Ries, J. (2020). SMAP: a modular super-resolution microscopy analysis platform for SMLM data. Nature Methods $17,870-872$.

Saff, E.B., and Kuijlaars, A.B.J. (1997). Distributing many points on a sphere. The Mathematical Intelligencer 19, 5-11.

Scott, B.L., Sochacki, K.A., Low-Nam, S.T., Bailey, E.M., Luu, Q., Hor, A., Dickey, A.M., Smith, S., Kerkvliet, J.G., Taraska, J.W., et al. (2018). Membrane bending occurs at all stages of clathrin-coat assembly and defines endocytic dynamics. Nature Communications 9, 419.

Smith, C.J., Grigorieff, N., and Pearse, B.M.F. (1998). Clathrin coats at $21 \AA$ resolution: a cellular assembly designed to recycle multiple membrane receptors. The EMBO Journal 17, 4943-4953.

Sochacki, K.A., and Taraska, J.W. (2018). From Flat to Curved Clathrin : Controlling a Plastic Ratchet. Trends in Cell Biology 1-16.

Sochacki, K.A., Dickey, A.M., Strub, M.-P., and Taraska, J.W. (2017). Endocytic proteins are partitioned at the edge of the clathrin lattice in mammalian cells. Nature Cell Biology 12, 517.

Sochacki, K.A., Heine, B.L., Haber, G.J., Jimah, J.R., Prasai, B., Alfonzo-Méndez, M.A., Roberts, A.D., Somasundaram, A., Hinshaw, J.E., and Taraska, J.W. (2021). The structure and spontaneous curvature of clathrin lattices at the plasma membrane. Developmental Cell 56, 1131-1146.e3.

Tagiltsev, G., Haselwandter, C.A., and Scheuring, S. (2021). Nanodissected elastically loaded clathrin lattices relax to increased curvature. Sci Adv 7, eabg9934.

Takei, K., Haucke, V., Slepnev, V., Farsad, K., Salazar, M., Chen, H., and De Camilli, P. (1998). Generation of Coated Intermediates of Clathrin-Mediated Endocytosis on Protein-Free Liposomes. Cell 94, 131-141.

Taylor, M.J., Perrais, D., and Merrifield, C.J. (2011). A high precision survey of the molecular dynamics of mammalian clathrin-mediated endocytosis. PLoS Biology 9, e1000604.

Thevathasan, J.V., Kahnwald, M., Cieśliński, K., Hoess, P., Peneti, S.K., Reitberger, M., Heid, D., Kasuba, K.C., Hoerner, S.J., Li, Y., et al. (2019). Nuclear pores as versatile reference standards for quantitative superresolution microscopy. Nature Methods 16, 1045-1053.

Ungewickell, E., and Branton, D. (1981). Assembly units of clathrin coats. Nature 289, 420-422.

Willy, N.M., Ferguson, J.P., Silahli, S., Cakez, C., Hasan, F., Chang, H.C., Travesset, A., Li, S., Zandi, R., Li, D., et al. (2019). Endocytic Clathrin Coats Develop Curvature at Early Stages of Their Formation. BioRxiv 715219.

Wu, Y.-L., Tschanz, A., Krupnik, L., and Ries, J. (2020). Quantitative Data Analysis in Single-Molecule Localization Microscopy. Trends in Cell Biology 30, 837-851.

Wu, Y.-L., Hoess, P., Tschanz, A., Matti, U., Mund, M., and Ries, J. (2021). Maximum-likelihood model fitting for quantitative analysis of SMLM data. BioRxiv 2021.08.30.456756.

Yoshida, A., Sakai, N., Uekusa, Y., Imaoka, Y., Itagaki, Y., Suzuki, Y., and Yoshimura, S.H. (2018). Morphological changes of plasma membrane and protein assembly during clathrin-mediated endocytosis. PLOS Biology 16, e2004786.

Zeno, W.F., Hochfelder, J.B., Thatte, A.S., Wang, L., Gadok, A.K., Hayden, C.C., Lafer, E.M., and Stachowiak, J.C. (2021). Clathrin senses membrane curvature. Biophysical Journal 120, 818-828.

Zhao, W., Hanson, L., Lou, H.-Y., Akamatsu, M., Chowdary, P.D., Santoro, F., Marks, J.R., Grassart, A., Drubin, D.G., Cui, Y., et al. (2017). Nanoscale manipulation of membrane curvature for probing endocytosis in live cells. Nature Nanotechnology 11, 822. 
bioRxiv preprint doi: https://doi.org/10.1101/2021.10.12.463947; this version posted October 13, 2021. The copyright holder for this

preprint (which was not certified by peer review) is the author/funder, who has granted bioRxiv a license to display the preprint in perpetuity. It is made available under aCC-BY-NC-ND 4.0 International license.

\section{Supplementary Information}

(A) Clusters of clathrin structures

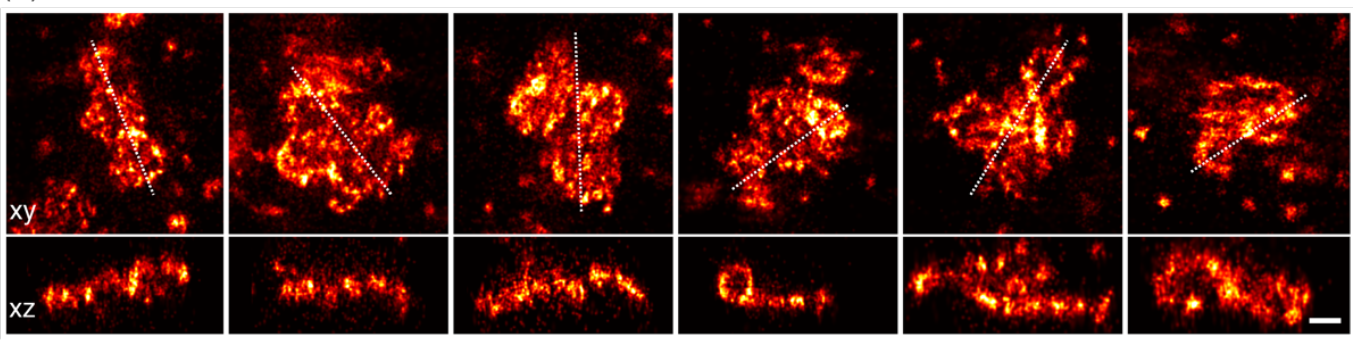

(B) Vesicular structures

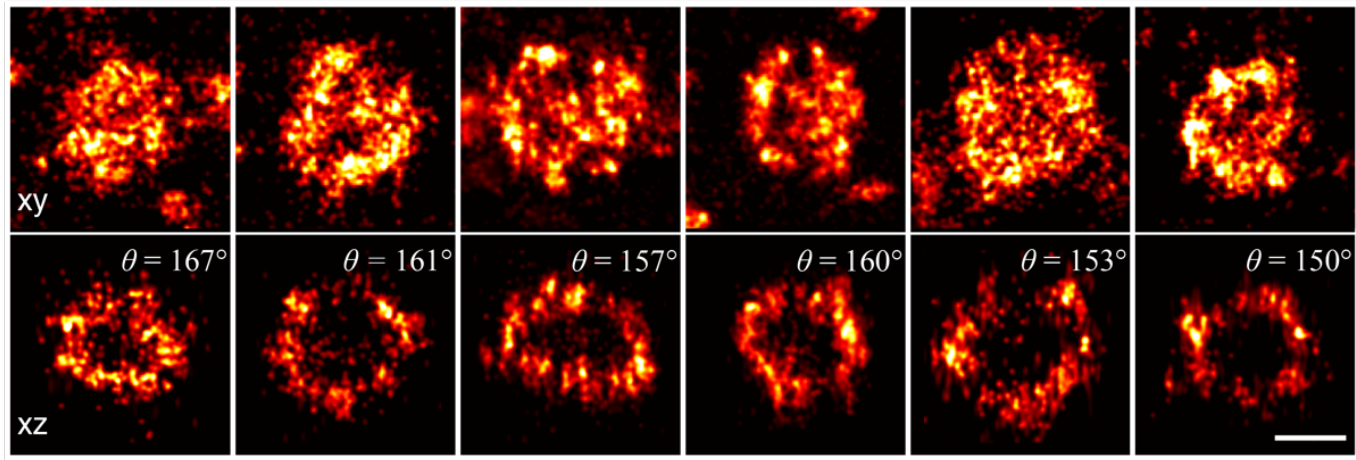

(C) Asymmetric structures

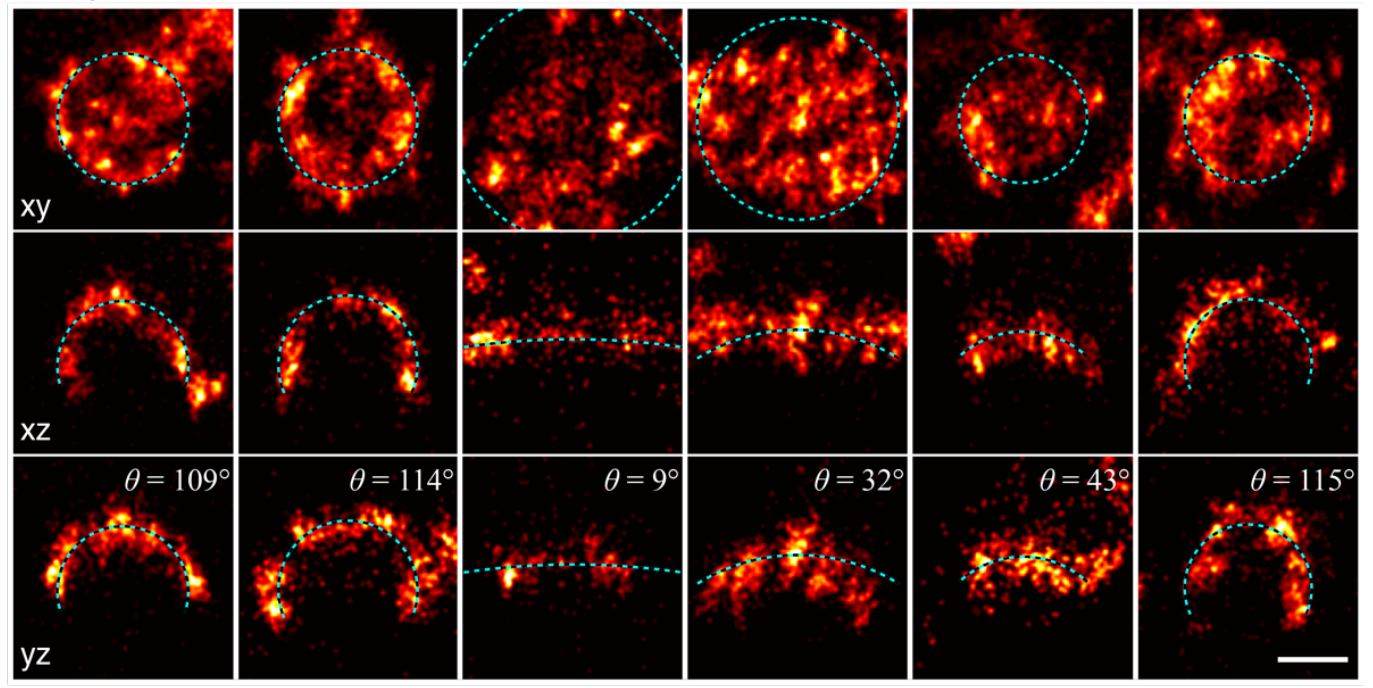

(D) Holes in clathrin coat labeling

\begin{tabular}{|c|c|c|c|c|c|}
\hline$x^{\infty}+4$ & 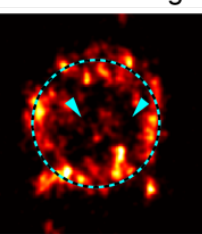 & 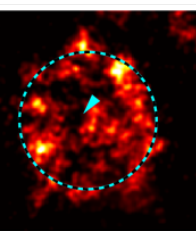 & $\left(\begin{array}{l}1 \\
2 \\
2\end{array}\right.$ & 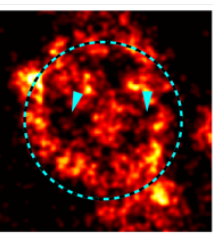 & int \\
\hline $\begin{array}{l}\theta=124^{\circ} \\
y^{2}\end{array}$ & ${ }^{\theta=119^{\circ}}$ & $\theta=83^{\circ}$ & $\begin{array}{r}\theta=80^{\circ} \\
x^{3}+y^{2}=0\end{array}$ & $\begin{array}{r}\theta=90^{\circ} \\
37\end{array}$ & $\begin{array}{l}\quad \theta=67 \\
\ddots \cdots\end{array}$ \\
\hline
\end{tabular}


bioRxiv preprint doi: https://doi.org/10.1101/2021.10.12.463947; this version posted October 13, 2021. The copyright holder for this preprint (which was not certified by peer review) is the author/funder, who has granted bioRxiv a license to display the preprint in perpetuity. It is made available under aCC-BY-NC-ND 4.0 International license.

Supplementary Figure 1 - Examples of diverse clathrin coat structures. (A) Large clusters of clathrin molecules excluded from further analysis. Shown in top view (xy) with dotted line indicating a $50 \mathrm{~nm}$-thick z-slice (xz) shown below. (B) Vesicular structures are sometimes fitted with a lower $\theta$ as expected. (C) While a spherical model describes structure of most endocytic clathrin coats faithfully, there are few cases, as exemplified here, where the elliptical and irregular shape of an assembling coat is difficult to approximate with a simple geometric model. Two orthogonal $50 \mathrm{~nm}$-thick z-slices are shown here in $x z$ and $y z$, and the respective spherical model fit is plotted as a dotted line. (D) Non-continuous labeling of clathrin manifests itself as holes in the coat, indicated with a blue arrow. All scale bars are $100 \mathrm{~nm}$. 
bioRxiv preprint doi: https://doi.org/10.1101/2021.10.12.463947; this version posted October 13, 2021. The copyright holder for this preprint (which was not certified by peer review) is the author/funder, who has granted bioRxiv a license to display the preprint in perpetuity. It is made available under aCC-BY-NC-ND 4.0 International license.
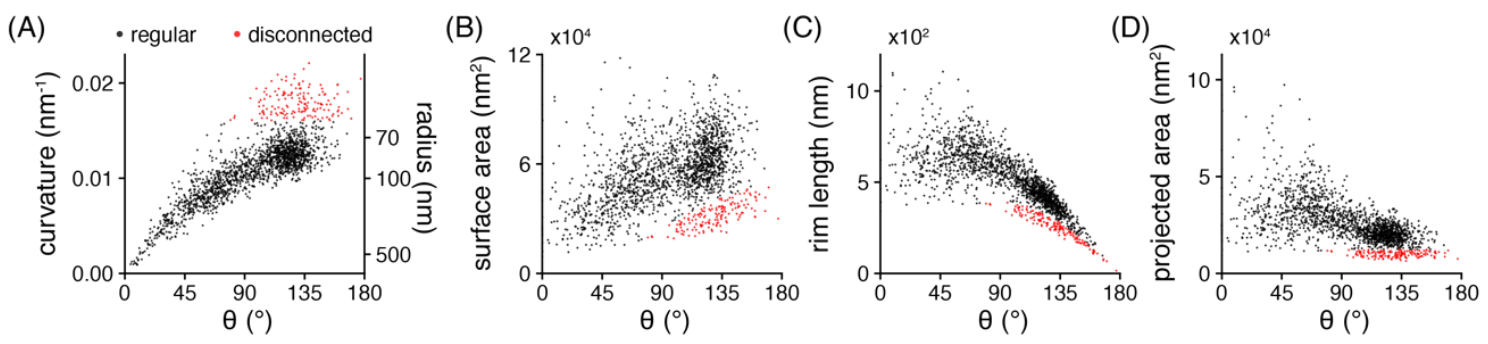

(B)
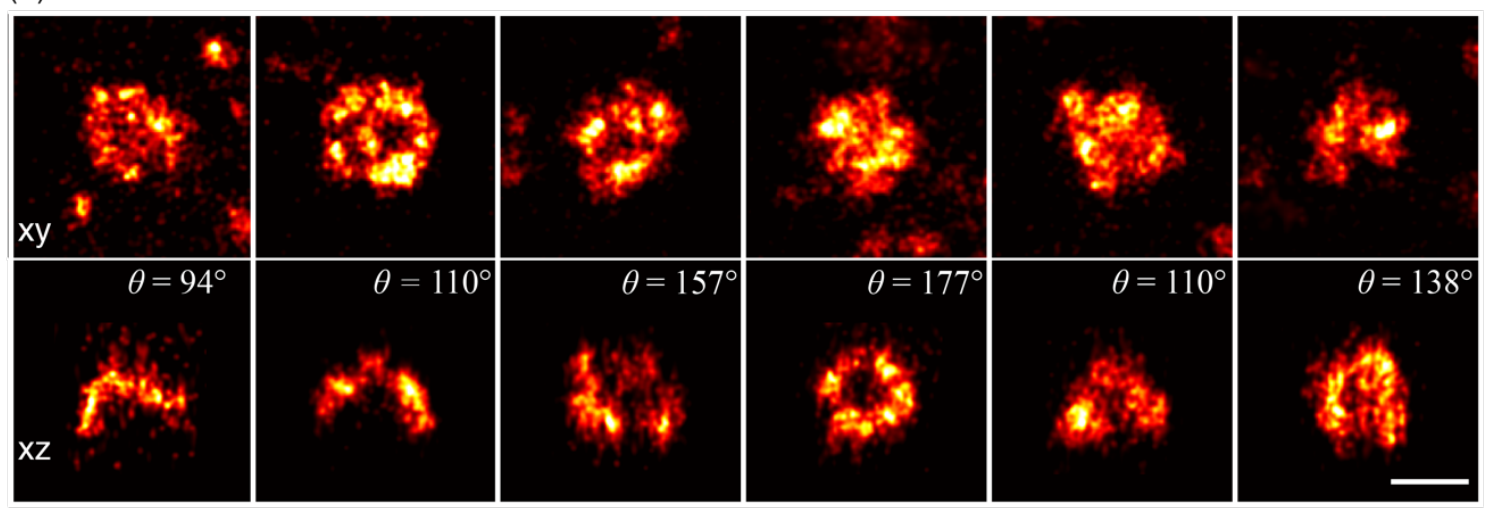

Supplementary Figure 2 - Non-endocytic clathrin structures. Results of LocMoFit analysis of clathrin structures in SK-MEL-2 cells ( $N=13$ cells, $n=1798$ sites). (A) A strong correlation between curvature and $\theta$ can be observed for most structures ( $n=1645$ sites, black). A disconnected point cloud ( $n=153$ sites, 8.5\%, red) indicates the presence of endocytosis-unrelated clathrin structures. The same distinct population of data points can be observed for the (B) surface area, (C) rim length, and (D) projected area. (B) Example structures from the disconnected population of sites in top view (xy) and $50 \mathrm{~nm}$-thick z-slices (xz) and their respective $\theta$ values. Scale bar is $100 \mathrm{~nm}$. 
(A) U2OS
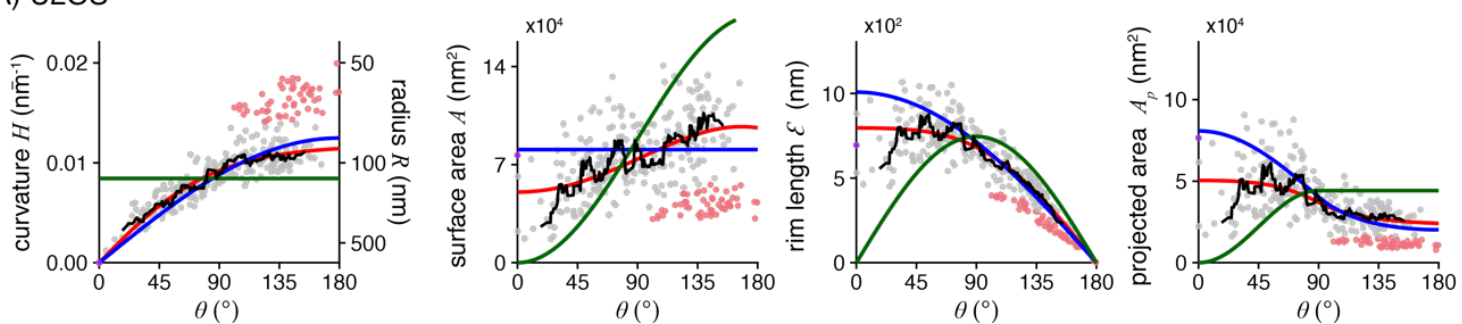

(B) 3 T3
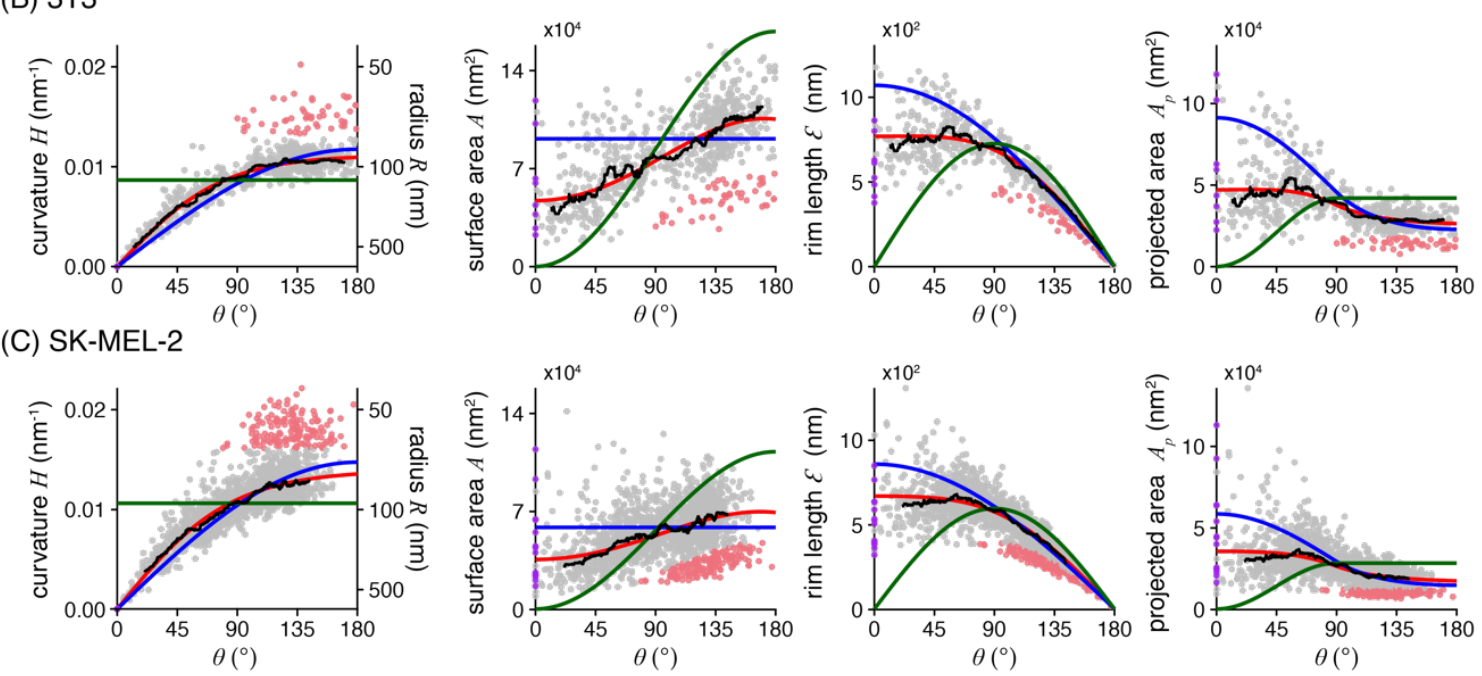

- cooperative curvature model - constant curvature model - constant area model - rolling median

- used for model fit - disconnected sites • flat sites

Supplementary Figure 3 - Clathrin coat growth modelled for three different cell lines. Results of LocMoFit analysis for clathrin structures. Different growth models and a rolling median (black, window width $=5 \%$ of total number of sites) are fitted to curvature over $\theta$ for the main data points (grey). The resulting fitting parameters are then used to map the same models also over surface area, rim length, and projected area (left to right). The disconnected sites (rose) and completely flat sites (curvature $=0 \mathrm{~nm}^{-1}$ and $\theta=0^{\circ}$, purple) were excluded from the fitting. This analysis was performed for clathrin structures imaged in (A) U2OS ( $N=1$ cell, 95 fitted sites, 17 disconnected sites, 0 completely flat sites), (B) $3 T 3$ mouse fibroblasts ( $N=7$ cells, $n_{\text {grey }}=680$ sites, $n=51$ disconnected sites, $n=8$ completely flat sites), and (C) SK-MEL-2 cells ( $N=13$ cells, $n=1631$ fitted sites, $n=153$ disconnected sites, $n=$ completely flat sites ). 

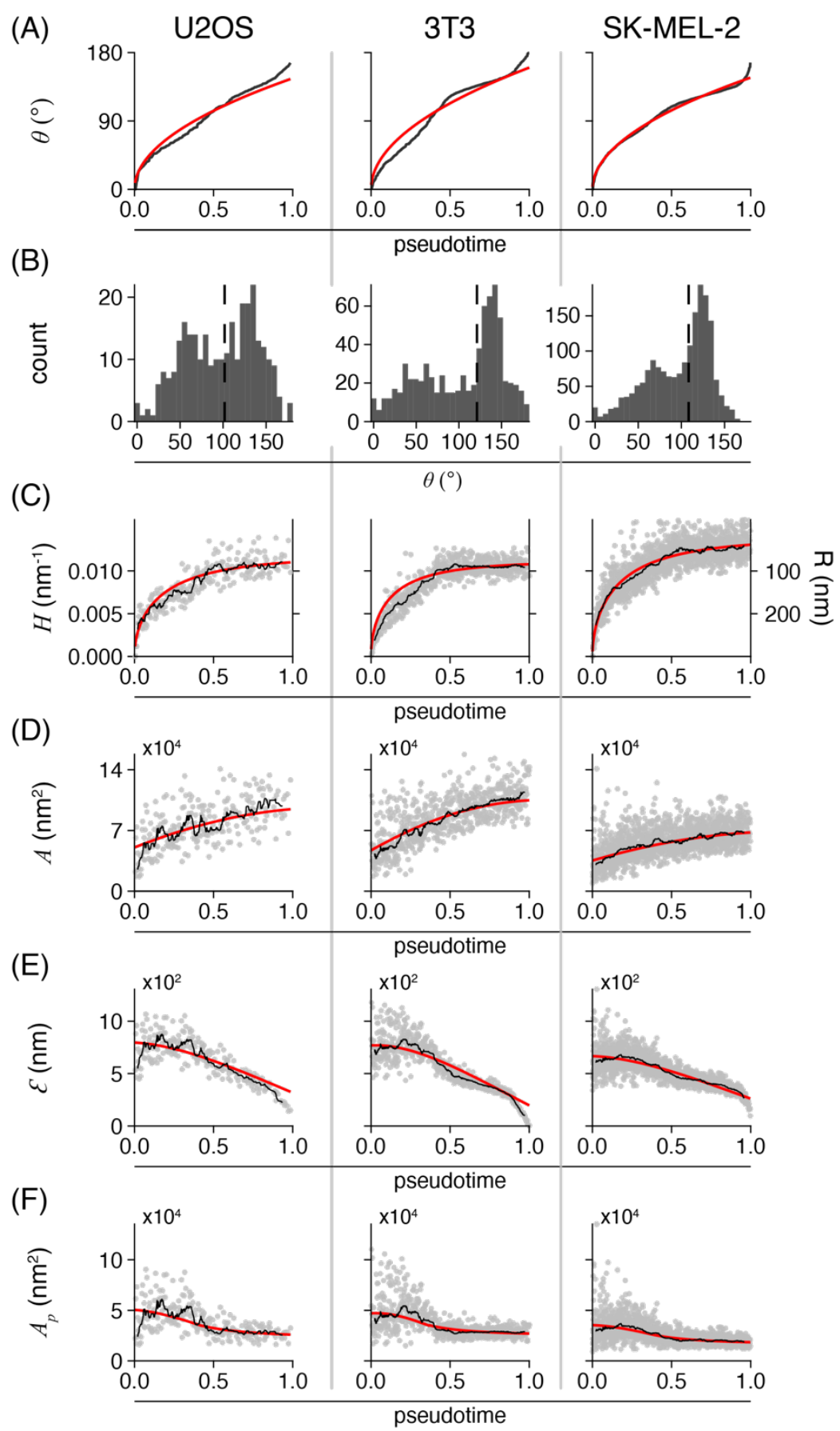

Supplementary Figure 4 - Temporal reconstruction of clathrin coat remodeling in three cell lines. U2OS, $3 T 3$ mouse fibroblasts and SK-MEL-2 cells (from left to right). (A) The cooperative curvature model (red line) highlights the square-root dependence between $\theta$ and pseudotime. (B) Cell line specific distribution of $\theta$ show slight differences in their enrichments, especially in the earlier states. Median $\theta$ shown as dotted lines correspond to $99.6^{\circ}$ for U2OS; $121.4^{\circ}$ for $3 T 3$; and $108.5^{\circ}$ for SK-MEL-2 cells. The cooperative curvature model is used to describe the (C) curvature $H$ propagation over pseudotime. Resulting fitting parameters are then used to map the same model to $(D)$ surface area $A,(E)$ rim length $\varepsilon$ and $(F)$ projected area $A_{p}$. A rolling median is plotted for $(C)-(F)$ in black (window width $=5 \%$ of total number of sites). 
(A)

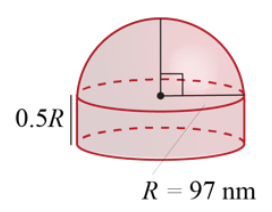

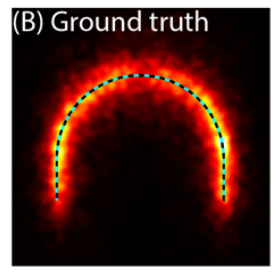

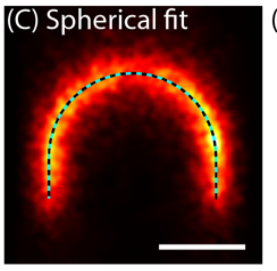

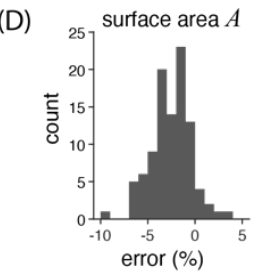

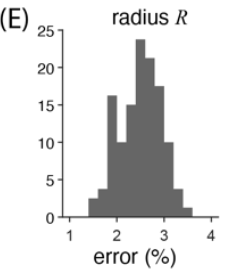

Supplementary Figure 5 - Averaging preserves U-shapes in simulated endocytic sites. (A) A ground-truth structure was used for simulating U-shaped clathrin coats. The model for simulation was built by combining a hemisphere with a radius of $R=97 \mathrm{~nm}$ and a cylinder with the height $D=0.5 R$. We choose the radius value according to the median radius of bin 6 in Figure $4 G$. This bin has a closing angle slightly larger than $90^{\circ}$ and hence could be where the U-shaped clathrin coats are most likely to be sorted to if they exist. 20-nm thick crosssections of the averages ( $N=100$ sites) registered based on (B) the ground truth and (C) the spherical fit are shown with the ground-truth U-shaped model (dotted line). Histograms of normalized estimation errors are shown for the parameters $(D)$ surface area and $(E)$ radius, with mean values of $-2.6 \%$ and $2.5 \%$ respectively. The scale bar is $100 \mathrm{~nm}$. 
Table 1 - Summary of clathrin coat growth model fits in SK-MEL-2. Fitted parameter values for the constant area model (CAM), the constant curvature model (CCM) and the cooperative curvature model (CoopCM) when fitting curvature $H(\theta)$, surface area $A(\theta)$, rim length $\varepsilon(\theta)$ and projected area $A_{p}(\theta)$. A: Surface area fitted with CAM; R: Radius fitted with CCM; $\gamma$ : Constant rate of curvature increase fitted with CoopCM; $H_{0}$ : Preferred curvature of the clathrin coat fitted with CoopCM; $R_{0}$ : preferred radius of the clathrin coat fitted with CoopCM; $A_{0}$ : Fraction of surface area growing as a flat lattice before curvature initiation, defined as $A_{0}=A(\theta=$ $0.01) / A(\theta=\pi)$; $k_{o n}$ : local growth rate obtained from $\theta(t)$ fitted with CoopCM and measured in $\mathrm{nm}$ per pseudotime units $\tilde{s}^{-1}$.

\begin{tabular}{llllll} 
& & Curvature $\mathbf{H}(\boldsymbol{\theta})$ & Surface area $\mathbf{A}(\boldsymbol{\theta})$ & Rim length $\boldsymbol{\varepsilon}(\boldsymbol{\theta})$ & Projected area $\mathbf{A}_{\mathrm{p}}(\boldsymbol{\theta})$ \\
\hline CAM & $\mathbf{A}$ & $58300 \pm 400 \mathrm{~nm}^{2}$ & $56700 \pm 400 \mathrm{~nm}^{2}$ & $49600 \pm 500 \mathrm{~nm}^{2}$ & $48800 \pm 500 \mathrm{~nm}^{2}$ \\
\hline CCM & $\mathbf{R}$ & $94.5 \pm 0.6 \mathrm{~nm}$ & $84.8 \pm 0.4 \mathrm{~nm}$ & $97.3 \pm 0.8 \mathrm{~nm}$ & $92.8 \pm 0.7 \mathrm{~nm}$ \\
\hline CoopCM & $\boldsymbol{\gamma}$ & $(9.4 \pm 0.1) \cdot 10^{-3} \mathrm{~nm}^{-1}$ & $(9.0 \pm 0.1) \cdot 10^{-3} \mathrm{~nm}^{-1}$ & $(9.4 \pm 0.1) \cdot 10^{-3} \mathrm{~nm}^{-1}$ & $(9.2 \pm 0.1) \cdot 10^{-3} \mathrm{~nm}^{-1}$ \\
& $\mathbf{H}_{\mathbf{0}}$ & $(13.9 \pm 0.1) \cdot 10^{-3} \mathrm{~nm}^{-1}$ & $(13.8 \pm 0.1) \cdot 10^{-3} \mathrm{~nm}^{-1}$ & $(13.4 \pm 0.1) \cdot 10^{-3} \mathrm{~nm}^{-1}$ & $(13.5 \pm 0.1) \cdot 10^{-3} \mathrm{~nm}^{-1}$ \\
& $\mathbf{R}_{\mathbf{0}}$ & $72.0 \pm 0.6 \mathrm{~nm}$ & $72.2 \pm 0.6 \mathrm{~nm}$ & $74.3 \pm 0.8 \mathrm{~nm}$ & $73.9 \pm 0.8 \mathrm{~nm}$ \\
& $\mathbf{A}_{\mathbf{0}}$ & 0.51 & 0.55 & 0.48 & 0.51 \\
& $\mathbf{k}_{\text {on }} 78.1 \mathrm{~nm} \tilde{s}^{-1}$ & $73.2 \mathrm{~nm}^{-1}$ & $85.4 \mathrm{~nm}^{-1}$ & $80.5 \mathrm{~nm}^{-1}$
\end{tabular}

Table 2 - Summary of clathrin coat growth model fits in U2OS. Fitted parameter values for the constant area model (CAM), the constant curvature model (CCM) and the cooperative curvature model (CoopCM) when fitting curvature $H(\theta)$, surface area $A(\theta)$, rim length $\varepsilon(\theta)$ and projected area $A_{p}(\theta)$. A: Surface area fitted with $C A M$; $R$ : Radius fitted with CCM; $\gamma$ : Constant rate of curvature increase fitted with CoopCM; $\mathrm{H}_{0}$ : Preferred curvature of the clathrin coat fitted with CoopCM; Ro: preferred radius of the clathrin coat fitted with CoopCM; $A_{0}$ : Fraction of surface area growing as a flat lattice before curvature initiation, defined as $A_{0}=A(\theta=0.01) / A(\theta=\pi) ; k_{\text {on }}$ : local growth rate obtained from $\theta(t)$ fitted with CoopCM and measured in nm per pseudotime units $\tilde{s}^{-1}$.

\begin{tabular}{llllll} 
& & Curvature $\mathbf{H}(\boldsymbol{\theta})$ & Surface area $\mathbf{A}(\boldsymbol{\theta})$ & Rim length $\boldsymbol{\varepsilon}(\boldsymbol{\theta})$ & Projected area $\mathbf{A}_{\mathbf{p}}(\boldsymbol{\theta})$ \\
\hline CAM & $\mathbf{A}$ & $80775 \pm 1605 \mathrm{~nm}^{2}$ & $76875 \pm 1659 \mathrm{~nm}^{2}$ & $65771 \pm 1645 \mathrm{~nm}^{2}$ & $65261 \pm 1632 \mathrm{~nm}^{2}$ \\
\hline $\mathbf{C C M}$ & $\mathbf{R}$ & $118.6 \pm 2.4 \mathrm{~nm}$ & $101.6 \pm 1.5 \mathrm{~nm}$ & $122.8 \pm 2.6 \mathrm{~nm}$ & $115 \pm 2.3 \mathrm{~nm}$ \\
\hline CoopCM & $\boldsymbol{\gamma}$ & $(7.9 \pm 0.2) \cdot 10^{-3} \mathrm{~nm}^{-1}$ & $(7.7 \pm 0.2) \cdot 10^{-3} \mathrm{~nm}^{-1}$ & $(8.1 \pm 0.2) \cdot 10^{-3} \mathrm{~nm}^{-1}$ & $(8.0 \pm 0.2) \cdot 10^{-3} \mathrm{~nm}^{-1}$ \\
& $\mathbf{H}_{\mathbf{0}}$ & $(11.8 \pm 0.2) \cdot 10^{-3} \mathrm{~nm}^{-1}$ & $(11.5 \pm 0.2) \cdot 10^{-3} \mathrm{~nm}^{-1}$ & $(11.0 \pm 0.3) \cdot 10^{-3} \mathrm{~nm}^{-1}$ & $(11.0 \pm 0.3) \cdot 10^{-3} \mathrm{~nm}^{-1}$ \\
& $\mathbf{R}_{\mathbf{0}}$ & $85.0 \pm 1.8 \mathrm{~nm}$ & $87.1 \pm 1.7 \mathrm{~nm}$ & $91.1 \pm 2.7 \mathrm{~nm}$ & $90.8 \pm 2.6 \mathrm{~nm}$ \\
& $\mathbf{A}_{\mathbf{0}}$ & 0.52 & 0.52 & 0.44 & 0.46 \\
& $\mathbf{k}_{\text {on }}$ & $89.3 \mathrm{~nm} \tilde{s}^{-1}$ & $92.4 \mathrm{~nm}^{-1}$ & $113.3 \mathrm{~nm} \tilde{s}^{-1}$ & $108.2 \mathrm{~nm}^{-1}$
\end{tabular}

Table 3-Summary of clathrin coat growth model fits in 3T3 mouse fibroblasts. Fitted parameter values for the constant area model (CAM), the constant curvature model (CCM) and the cooperative curvature model (CoopCM) when fitting curvature $H(\theta)$, surface area $A(\theta)$, rim length $\varepsilon(\theta)$ and projected area $A_{p}(\theta)$. A: Surface area fitted with CAM; R: Radius fitted with CCM; $\gamma$ : Constant rate of curvature increase fitted with CoopCM; $H_{0}$ : Preferred curvature of the clathrin coat fitted with CoopCM; $R_{0}$ : preferred radius of the clathrin coat fitted with CoopCM; $A_{0}$ : Fraction of surface area growing as a flat lattice before curvature initiation, defined as $A_{0}=A(\theta=$ $0.01) / A(\theta=\pi)$; $k_{o n}$ : local growth rate obtained from $\theta(t)$ fitted with CoopCM and measured in $\mathrm{nm}$ per pseudotime units $\tilde{s}^{-1}$.

\begin{tabular}{llllll} 
& & Curvature $\mathbf{H}(\boldsymbol{\theta})$ & Surface area $\mathbf{A}(\boldsymbol{\theta})$ & Rim length $\boldsymbol{\varepsilon}(\boldsymbol{\theta})$ & Projected area $\mathbf{A}_{\mathbf{p}}(\boldsymbol{\theta})$ \\
\hline CAM & $\mathbf{A}$ & $91200 \pm 1000 \mathrm{~nm}^{2}$ & $84500 \pm 1000 \mathrm{~nm}^{2}$ & $66200 \pm 1000 \mathrm{~nm}^{2}$ & $66500 \pm 1000 \mathrm{~nm}^{2}$ \\
\hline CCM & $\mathbf{R}$ & $115.6 \pm 1.4 \mathrm{~nm}$ & $98.8 \pm 0.7 \mathrm{~nm}$ & $121.9 \pm 1.9 \mathrm{~nm}$ & $109.1 \pm 1.5 \mathrm{~nm}$ \\
\hline CoopCM & $\boldsymbol{\gamma}$ & $(8.2 \pm 0.1) \cdot 10^{-3} \mathrm{~nm}^{-1}$ & $(7.8 \pm 0.1) \cdot 10^{-3} \mathrm{~nm}^{-1}$ & $(8.0 \pm 0.1) \cdot 10^{-3} \mathrm{~nm}^{-1}$ & $(7.8 \pm 0.1) \cdot 10^{-3} \mathrm{~nm}^{-1}$ \\
& $\mathbf{H}_{\mathbf{0}}$ & $(11.2 \pm 0.1) \cdot 10^{-3} \mathrm{~nm}^{-1}$ & $(11.1 \pm 0.1) \cdot 10^{-3} \mathrm{~nm}^{-1}$ & $(11.1 \pm 0.2) \cdot 10^{-3} \mathrm{~nm}^{-1}$ & $(11.1 \pm 0.1) \cdot 10^{-3} \mathrm{~nm}^{-1}$ \\
& $\mathbf{R}_{\mathbf{0}}$ & $89.7 \pm 0.7 \mathrm{~nm}$ & $90.0 \pm 0.7 \mathrm{~nm}$ & $90.2 \pm 1.4 \mathrm{~nm}$ & $90.4 \pm 1.2 \mathrm{~nm}$ \\
& $\mathbf{A}_{\mathbf{0}}$ & 0.45 & 0.48 & 0.46 & 0.48 \\
& $\mathbf{k}_{\text {on }}$ & $131.3 \mathrm{~nm} \tilde{s}^{-1}$ & $123.9 \mathrm{~nm}^{-1}$ & $128.6 \mathrm{~nm}^{-1}$ & $125.4 \mathrm{~nm}^{-1}$
\end{tabular}




\title{
Supplemental note to: Superresolution microscopy reveals partial preassembly and subsequent bending of the clathrin coat during endocytosis
}

\author{
Markus Mund, Aline Tschanz, Yu-Le Wu, Felix Frey, Johanna Mehl, \\ Marko Kaksonen, Ori Avinoam, Ulrich S. Schwarz, and Jonas Ries
}

\section{COOPERATIVE CURVATURE MODEL}

We derive a kinetic model for clathrin-mediated endocytosis that is based on minimal assumptions based on our experimental observations. We first assume that the area $A$ of clathrin coats grows mainly by addition of new triskelia at the edges [1]. With a local growth rate $k_{\text {on }}$ and an edge length $\mathcal{E}$, we have the simple growth law

$$
\frac{\mathrm{d}}{\mathrm{d} t} A=\dot{A}=k_{\text {on }} \mathcal{E} \text {. }
$$

We also know from our experimental observations that all clathrin-coated pits have the geometry of a spherical cap with area $A_{\text {cap }}=2 \pi R^{2}(1-\cos \theta)$, where $R$ is the cap radius and $\theta$ the closing angle. The edge length then is $\mathcal{E}_{\text {cap }}=2 \pi R \sin \theta$. Using these formulas on Eq. (1) we find

$$
2 \dot{R} \tan \frac{\theta}{2}+R \dot{\theta}=k_{\text {on }} .
$$

Because we parametrize the shape of the growing clathrin coat by the two dynamical variables $R(t)$ and $\theta(t)$, but have only one growth equation up to now, we have to make additional assumptions to completely define our kinetic model.

As the main function of clathrin is to generate curvature, we now focus on coat curvature $H=1 / R$ and assume that it increases with a basal rate $\gamma$. Experimentally we observe that clathrin patches first grow flat and then start to curve. Curvature generation in clathrin lattices is the combination of a preferred curvature of the single triskelion and cooperative effects in the lattice $[2,3]$. Several mechanisms exist that might generate curvature during coat growth. First, clathrin triskelia are likely to be geometrically frustrated within the flat clathrin coat. To overcome this frustration, they would have to adjust their positions relative to their neighbors, such that their free energy is getting more favorable. Second, lattice vacancies could be filled up with new triskelia, as predicted theoretically [4]. Note that these vacancies would not necessarily relate to patch area, because the clathrin lattice consists of many overlapping arms. Filling of vacancies would however increase clathrin density and coat stiffness, and thus could also drive invagination of the coat [5]. We note that it was indeed confirmed experimentally that the clathrin density increases within the coat during invagination $[2,6]$. Third, lattice pentagons, associated with lattice curvature, could form at the edge of the clathrin lattice and diffuse into the coat, similar to lattice defects on curved surfaces [7]. All three mechanisms could generate curvature with a certain rate which we assume to be constant during the initial stages of growth.

At late stages, increase of curvature has to stop and therefore we assume curvature saturation at a characteristic value $H_{0}$, which is similar to, but different from the radius measured for clathrin cages. A simple estimate would be $40 \mathrm{~nm}$ for a typical radius of the membranes in the pits [8] plus $15 \mathrm{~nm}$ thickness of the clathrin coat [9]. These $55 \mathrm{~nm}$ would be larger than the $40 \mathrm{~nm}$ for clathrin cages, which are expected to be frustrated. If all triskelia in the lattice had achieved their optimal positions, a preferred or spontaneous curvature $H_{0}$ should emerge. Thus a growth equation for $H$ should have a stable fixed point generated by a higher order term. Since the corresponding mechanism is known to be of cooperative nature, larger groups of triskelia should be involved and the mechanism should start to dominate relatively late in the process, but then dominate quickly. Here we assume that the mechanism is proportional to $H^{2}$ (rather than to $H$, with a linear law corresponding to a non-cooperative effect that starts to show early). Assuming that curvature generation is a geometrical effect, its time development should depend on the closing angle and we get

$$
\frac{\mathrm{d} H}{\mathrm{~d} \theta}=\gamma\left(1-\frac{H^{2}}{H_{0}^{2}}\right) .
$$

The stable fixed point at $H=H_{0}$ can then be interpreted as corresponding to the preferred curvature of the mature coat.

Eq. (3) can be solved with the initial condition $H(\theta=0)=0$ by

$$
H(\theta)=H_{0} \tanh \left(\frac{\gamma}{H_{0}} \theta\right)
$$


TABLE I. Coat curvature, coat area, and edge length summarized for the CCM, the CAM, and the CoopCM.

\begin{tabular}{llll}
\hline & $\mathrm{CCM}$ & $\mathrm{CAM}$ & $\mathrm{CoopCM}$ \\
\hline$H$ & $1 / R_{\mathrm{CCM}}$ & $\sqrt{\frac{2 \pi(1-\cos \theta)}{A_{\mathrm{CAM}}}}$ & $1 / R_{\text {coat }}$ \\
$A$ & $2 \pi R_{\mathrm{CCM}}^{2}(1-\cos \theta)$ & $A_{\mathrm{CAM}}$ & $2 \pi R_{\text {coat }}^{2}(1-\cos \theta)$ \\
$\mathcal{E}$ & $2 \pi R_{\mathrm{CCM}} \sin \theta$ & $\sqrt{\frac{2 \pi A_{\mathrm{CAM}}}{(1-\cos \theta)}} \sin \theta$ & $2 \pi R_{\text {coat }} \sin \theta$ \\
\hline
\end{tabular}

and defines the coat radius as a function of the closing angle

$$
R_{\text {coat }}=R(\theta)=\frac{1}{H(\theta)}=R_{0} \frac{1}{\tanh \left(\gamma R_{0} \theta\right)},
$$

with $R_{0}=1 / H_{0}$. We note that the expansion of Eq. (5) gives $R \propto 1 / \theta$ in leading order around the flat state.

We can combine Eq. (2) and Eq. (3) to find the full dynamics of coat invagination. Therefore, we compute the derivative of $R_{\text {coat }}$

$$
\frac{\mathrm{d} R_{\text {coat }}}{\mathrm{d} \theta}=-\frac{\gamma R_{0}^{2}}{\sinh ^{2}\left(\gamma R_{0} \theta\right)}
$$

We use Eq. (5) and Eq. (6) and the chain rule $\mathrm{d} R / \mathrm{d} t=\mathrm{d} R / \mathrm{d} \theta \mathrm{d} \theta / \mathrm{d} t$ to rewrite Eq. (2). Solving for $\dot{\theta}$ yields

$$
\dot{\theta}=k_{\mathrm{on}} \frac{1}{2 \frac{\mathrm{d} R(\theta)}{\mathrm{d} \theta} \tan \frac{\theta}{2}+R(\theta)} .
$$

After expanding Eq. (7) up to leading order in $\theta$ we find

$$
\dot{\theta}=\frac{12 \gamma k_{\mathrm{on}}}{8 \gamma^{2} R_{0}^{2}-1} \frac{1}{\theta}
$$

which is solved with the initial condition $\theta(t=0)=0$ by

$$
\theta(t)=\sqrt{\frac{24 \gamma k_{\mathrm{on}}}{8 \gamma^{2} R_{0}^{2}-1} t} .
$$

Thus the coat initially is flat $(\theta=0)$ and then starts to generate curvature, increasing the closing angle with a square root dependence in time, which reflects the slowing down of curvature generation as preferred curvature is approached. We refer to this model as the cooperative curvature model (CoopCM) due to the assumption of a non-linear growth law.

\section{FIT RESULTS}

To contrast the CoopCM with existing models, we fit it to experimental data for $H(\theta), A(\theta)$ and $\mathcal{E}(\theta)$. We compare the fitted curves to the constant curvature model (CCM), defined through the constant coat curvature $1 / R_{\mathrm{CCM}}$, and to the constant area model $(\mathrm{CAM})$, defined through the constant coat area $A_{\mathrm{CAM}}$. The equations for the curvature $H(\theta)=1 / R(\theta)$, the area $A(\theta)$, and the edge length $\mathcal{E}(\theta)$ are summarized in Table I for all three models. In order to fit, we first filter the data according to curvature $H$, as described in the methods section of the main text. We then fit the different models to each of the data sets separately. For the CCM and CAM we get values for one parameter each, namely $R_{\mathrm{CCM}}$ and $A_{\mathrm{CAM}}$, respectively. For the CoopCM we obtain two parameter values $\gamma$ and $H_{0}$. For the CoopCM we also determine the area when invagination occurs $A_{0}=A(\theta=0.01) / A(\theta=\pi)$, which is the relative transition size where the flat-to-curved-transition occurs. By definition $A_{\mathrm{FTC}}=0$ for the CCM, whereas $A_{\mathrm{FTC}}=1$ for the CAM. Moreover, for the CoopCM we also compute $R_{0}$. For all parameters we also determine the relative fit errors, given by $\sigma_{x} / x$, where $\sigma_{x}$ is the standard deviation of the parameter $x$ and $x$ is the value of the parameter. This procedure allows us to compare the fit results to each other. The fitted parameter values for the different models and cell lines are summarized in the Supplementary Tables 1-3.

We then fit Eq. (9) to the data, describing $\theta(t)$. Using the values of $\gamma$ and $H_{0}$, we determine $k_{\text {on }}$ from the fit. The resulting values, measured in units of the pseudo time $\tilde{s}$, are summarized in the Supplementary Tables 1-3. We do 
not show the fit errors of $k_{\text {on }}$, because they are small and result from the same fit of $\theta(t)$ and the errors of $\gamma$ and $H_{0}$, which does not allow us to discriminate between the different fits.

[1] D. Bucher, F. Frey, K. A. Sochacki, S. Kummer, J.-P. Bergeest, W. J. Godinez, H.-G. Kräusslich, K. Rohr, J. W. Taraska, U. S. Schwarz, et al., Clathrin-adaptor ratio and membrane tension regulate the flat-to-curved transition of the clathrin coat during endocytosis, Nat. Commun. 9, 1109 (2018).

[2] W. F. Zeno, J. B. Hochfelder, A. S. Thatte, L. Wang, A. K. Gadok, C. C. Hayden, E. M. Lafer, and J. C. Stachowiak, Clathrin senses membrane curvature, Biophys. J., S0006349521000801 (2021).

[3] K. A. Sochacki, B. L. Heine, G. J. Haber, J. R. Jimah, B. Prasai, M. A. Alfonzo-Mendez, A. D. Roberts, A. Somasundaram, J. E. Hinshaw, and J. W. Taraska, The structure and spontaneous curvature of clathrin lattices at the plasma membrane, bioRxiv 10.1101/2020.07.18.207258 (2020).

[4] F. Frey, D. Bucher, K. A. Sochacki, J. W. Taraska, S. Boulant, and U. S. Schwarz, Eden growth models for flat clathrin lattices with vacancies, New J. Phys. 22, 073043 (2020).

[5] F. Frey and U. S. Schwarz, Competing pathways for the invagination of clathrin-coated membranes, Soft Matter 16, 10723 (2020).

[6] K. A. Sochacki, A. M. Dickey, M.-P. Strub, and J. W. Taraska, Endocytic proteins are partitioned at the edge of the clathrin lattice in mammalian cells, Nat. Cell Biol. 19, 352-361 (2017).

[7] S. Agarwal and S. Hilgenfeldt, Simple, General Criterion for Onset of Disclination Disorder on Curved Surfaces, Phys. Rev. Lett. 125, 078003 (2020).

[8] O. Avinoam, M. Schorb, C. J. Beese, J. A. Briggs, and M. Kaksonen, Endocytic sites mature by continuous bending and remodeling of the clathrin coat, Science 348, 1369 (2015).

[9] M. Saleem, S. Morlot, A. Hohendahl, J. Manzi, M. Lenz, and A. Roux, A balance between membrane elasticity and polymerization energy sets the shape of spherical clathrin coats, Nat. Commun. 6, 6249 (2015). 